\title{
Pass-Through del Tipo de Cambio y del Salario: TeOría Y EVIDENCIA PARA LA INDUSTRIA MANUFACTURERA EN MÉXICO
}

\author{
Andrés Conesa Labastida
}

Septiembre de 1998

Documento de Investigación No. 9803

Dirección General de Investigación Económica

BANCO DE MÉXICO 


\title{
Pass-Through del Tipo de Cambio y del Salario:

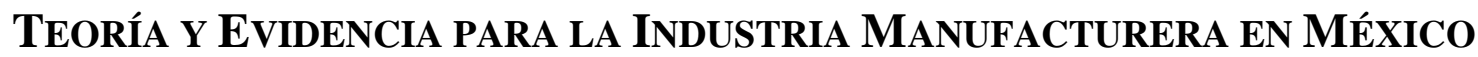

\author{
Andrés Conesa Labastida*
}

BANCO DE MÉXICO**

Septiembre de 1998

Documento de Investigación No. 9803

\begin{abstract}
RESUMEN
En este artículo se extienden los modelos tradicionales de pass-through al permitir que las empresas domésticas utilicen insumos importados. Esto crea un canal adicional por medio del cual el tipo de cambio puede afectar el nivel de precios. En términos de precios medidos en moneda extranjera, el modelo predice que el pass-through del tipo de cambio es mayor al pass-through de los salarios. Esta diferencia resulta menor en las industrias más competitivas, y es independiente del uso relativo de insumos importados por parte de las empresas domésticas. Estas predicciones son robustas para cualquier especificación tecnológica $\mathrm{y}$, bajo ciertas condiciones, para cualquier función de la demanda por el producto final.
\end{abstract}

Se comprueba la teoría utilizando información de 47 sectores de la industria manufacturera mexicana. Explotando la relación de cointegración (de largo plazo) entre las variables, se llevó a cabo un análisis empírico utilizando un modelo de corrección de errores. Las estimaciones de corto plazo coincidieron con las predicciones teóricas, mientras que los resultados de largo plazo sugieren que no existen diferencias significativas entre las elasticidades tipo de cambio y salario de los precios de equilibrio.

\footnotetext{
* Este trabajo fue elaborado cuando el autor se desempeñó como Investigador Económico en la Dirección de Estudios Económicos del Banco de México en el verano de 1996.

El autor agradece los comentarios de Guillermo Babatz, Demian Castillo, Gerardo Esquivel, Alexis Milo, Lorenza Martínez, Moisés Schwartz y Alejandro Werner.

${ }^{* *}$ El contenido de este documento no refleja necesariamente la opinión del Banco de México.
} 


\section{Contenido}

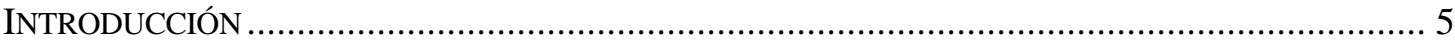

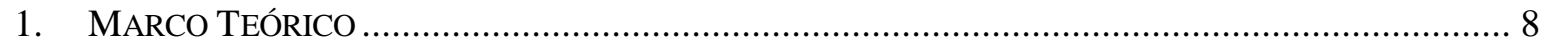

2. MARCO ECONÓMICO EN MÉXICO .............................................................................. 16

2.1 Tipo de Cambio, Salarios y Precios Internos ....................................................16

2.2 La Industria Manufacturera en México …............................................................18

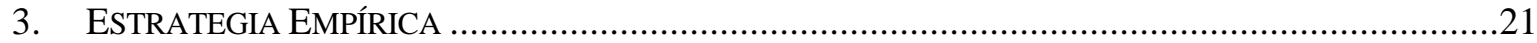

3.1 Pruebas de Estacionareidad y Cointegración ...........................................................21

3.2 Representación del Modelo de Corrección de Errores ...................................................23

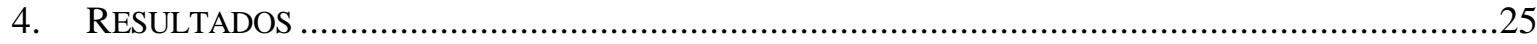

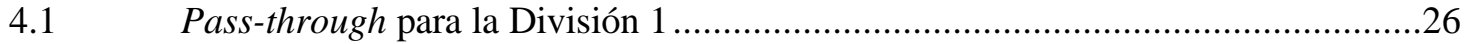

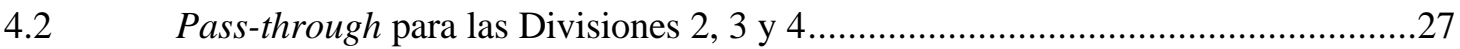

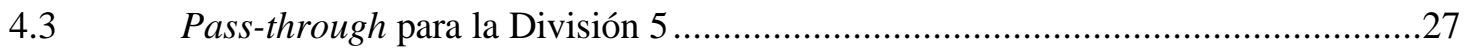

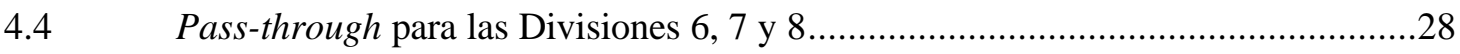

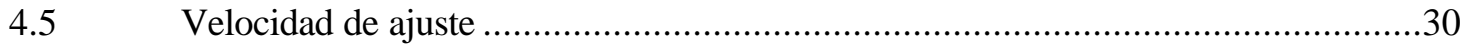

4.6 Pass-through, concentración de la Industria e Insumos Importados ......................32

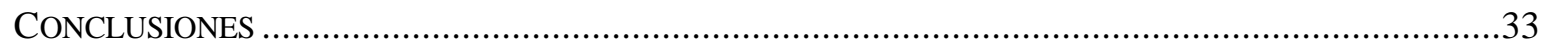

APÉNDICE A. ElASTICIDADES PRECIO Y MARKUPS DE EQUILIBRIO ..............................................34

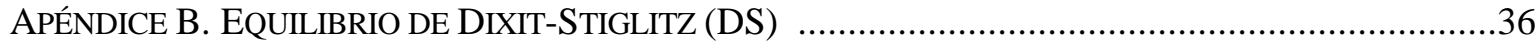

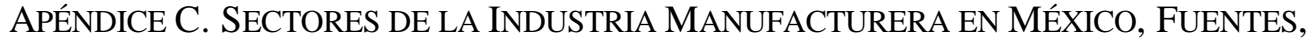

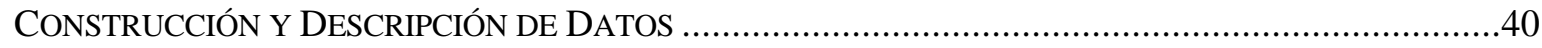

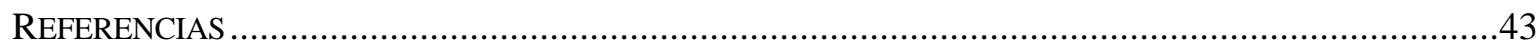




\section{Lista de Gráficas}

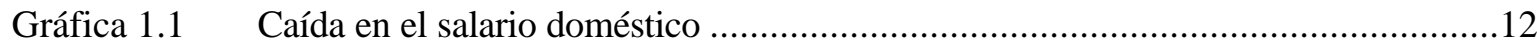

Gráfica 1.2 Aumento en el tipo de cambio nominal...........................................................13

Gráfica 2.1 Cambios en el tipo de cambio real mexicano ..................................................17

Gráfica 2.2 Índice de precios al consumidor de México en dólares .........................................18

Gráfica $4.1 \quad$ Elasticidades estimadas de largo plazo.......................................................25

Gráfica $4.2 \quad$ Elasticidades estimadas de corto plazo..........................................................

\section{Lista de Cuadros}

Cuadro 2.1 Uso de insumos importados en la Industria Manufacturera en México...................19

Cuadro 2.2 Intensidad de mano de obra en la Industria Manufacturera por división .................20

Cuadro 2.3 Razones de concentración en la Industria Manufacturera en México......................20

Cuadro 3.1 Pruebas de estacionareidad y cointegración......................................................22

Cuadro 4.1 Elasticidades estimadas de corto plazo para la industria de productos

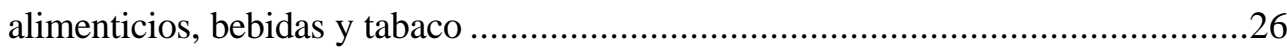

Cuadro 4.2 Elasticidades estimadas de corto plazo para las divisiones 2, 3 y 4 ...................27

Cuadro 4.3 Elasticidades estimadas de corto plazo para la industria de

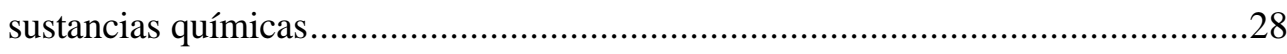

Cuadro 4.4 Elasticidades estimadas de corto plazo para las divisiones 6,7 y 8 ...................29

Cuadro 4.5 Número de periodos necesarios para alcanzar un determinado porcentaje de ajuste

Cuadro 4.6 Pass-through a precios en dólares, concentración e insumos importados ...............32

Cuadro $4.7 \quad$ Pass-through de precios en pesos e insumos importados ....................................32 


\section{Pass-Through Del Tipo De Cambio Y Del Salario: Teoría Y Evidencia Para La Industria Manufacturera En MéXico}

\section{Introducción}

La inflación es una de las principales preocupaciones de los instrumentadores de política económica en México. Después de haber experimentado bajos niveles de inflación y altas tasas de crecimiento durante los 25 años posteriores a la segunda guerra mundial, la tasa de inflación en México alcanzó cifras de dos dígitos en 1973. En 1987, cuando los precios crecieron a una tasa de $150 \%$, las autoridades decidieron lanzar un agresivo programa de estabilización, el cual incluía tanto reformas fiscales como políticas de ingresos (income policies). En este programa, el tipo de cambio actuó como ancla nominal para romper la inercia inflacionaria. Además, a través de una serie de pactos que frenaron la tasa de depreciación de la moneda en relación al movimiento del salario, en 1993 el gobierno logró reducir la inflación a niveles de un solo dígito por primera vez en veinte años. Durante este proceso, el tipo de cambio real (TCR) se apreció de manera constante, lo cual a su vez generó una pérdida en competitividad, y actuó como un freno del crecimiento, proceso que culminó en el colapso del tipo de cambio nominal de finales de 1994.

A pesar de que eventualmente las políticas de tipo de cambio y fijación de precios adoptadas en México se tradujeron en menores niveles de inflación, no había una clara comprensión acerca de la eficiencia relativa de cada uno de estas dos políticas. A priori, no es claro que una política de mantener la tasa de depreciación del tipo de cambio por debajo del incremento permitido en el salario, sea más eficiente para reducir la inflación que la política alternativa de sujetar los incrementos salariales por debajo de la tasa de depreciación cambiaria. Para estimar la eficiencia relativa de estas dos alternativas, necesitamos entender la manera en que los movimientos en el salario y en el tipo de cambio se traducen en cambios de precios. ¿Aumentan los precios en la misma magnitud con una depreciación del tipo de cambio que con un incremento proporcional en el salario? ¿Cómo cambia la respuesta a esta pregunta si la concentración industrial es distinta? ¿Es mayor el impacto de un movimiento del tipo de cambio sobre los precios si las empresas domésticas utilizan insumos importados? 
En la literatura económica se han escrito una cantidad considerable de estudios que intentan comprender el pass-through del tipo de cambio, es decir, la manera en que movimientos en el tipo de cambio se reflejan sobre los precios. De igual forma, muchos trabajos han analizado cómo el proceso de fijación de salarios se traduce en movimientos en el nivel de precios agregado, es decir, el passthrough del salario. En este contexto, el objetivo del presente artículo es juntar estas piezas en un solo modelo y estudiar conjuntamente cómo los movimientos en el tipo de cambio nominal y el salario afectan a los precios de la industria bajo ambientes competitivos alternativos. Además, se hace una extensión de los modelos tradicionales de pass-through del tipo de cambio ${ }^{1}$ al permitir que las empresas domésticas utilicen insumos importados, creando un canal adicional por medio del cual el tipo de cambio puede afectar el nivel de precios. Esta extensión es motivada por el hecho de que, comenzando con la liberalización comercial en 1985, las empresas mexicanas han incrementado su uso de mejores y más baratos insumos disponibles en los mercados internacionales.

Los argumentos teóricos en este artículo se basan en un modelo extendido de equilibrio de Cournot. La consistencia de los principales resultados es verificada analizando ambientes competitivos alternativos. Se encuentra que la elasticidad tipo de cambio y la elasticidad salario de los precios domésticos medidos en moneda doméstica $(p)$ son ambas menores a la unidad. Esto significa que los precios medidos en unidades de moneda extranjera $\left(p^{\mathrm{e}}\right)$ aumentarán si el salario doméstico aumenta, pero caerán después de una depreciación del tipo de cambio. Por lo tanto, la elasticidad de $p^{e}$ con respecto al tipo de cambio es negativa, mientras que la elasticidad con respecto al salario doméstico es positiva. A pesar de que este hallazgo es común en la literatura de modelos de pass-through, no ha sido enfatizado antes. Sin embargo, la principal contribución de este artículo es el demostrar no sólo que estas dos elasticidades tienen signo diferente, sino además que son de distinta magnitud. Específicamente, movimientos en el tipo de cambio nominal tendrán un mayor impacto sobre $p^{\mathrm{e}}$ que movimientos proporcionales en el salario nominal doméstico. Por ejemplo, los precios nacionales medidos en moneda extranjera deberán caer más después de una depreciación del tipo de cambio que después de una reducción proporcional en los salarios nominales ${ }^{2}$. Adicionalmente, se sugiere que este impacto asimétrico de los movimientos en el tipo de cambio y el salario sobre los precios es menor para industrias más competitivas e independiente del uso relativo de insumos importados por

1 Ver D ornbush (1987) para un análisis completo del passthraughen modelos de competencia imperfecta.

2 En otras palabras, la suma del passthraugh del tipo de cambio y del passthrough del salario hacia el precio, es menor a uno (e.g. la función de precios no es homogénea de grado uno en los precios de los factores). 
parte de las empresas domésticas. Los resultados son robustos a cualquier especificación tecnológica $\mathrm{y}$, bajo ciertas circunstancias, a cualquier función de demanda por el bien final ${ }^{3}$.

Estas predicciones teóricas se prueban utilizando datos de 47 sectores de la Industria Manufacturera en México. Explotando la relación de cointegración (de largo plazo) entre las variables, se llevó a cabo un análisis empírico utilizando un modelo de corrección de errores. Se encontró empíricamente que las elasticidades tipo de cambio y salario de corto plazo tienen los signos correctos. A excepción de cuatro sectores, se obtiene que el diferencial entre (el negativo de) la elasticidad tipo de cambio y la elasticidad salario doméstico es positiva. Este diferencial es estadísticamente significativo en 26 sectores, mayor para industrias más concentradas, e independiente del uso de insumos importados por parte de las empresas domésticas. También se encontró que el pass-through convencional del tipo de cambio hacia los precios en moneda doméstica es mayor en sectores que utilizan más intensivamente insumos importados y menos mano de obra doméstica. Estos sectores también presentan un menor pass-through del salario. Finalmente, en el largo plazo no se encontraron diferencias significativas entre el impacto del tipo de cambio y el salario sobre los precios.

Estos resultados sugieren que si el objetivo de la política económica es reducir los precios domésticos medidos en moneda extranjera, es más fácil lograrlo por medio de una devaluación del tipo de cambio nominal que con una reducción proporcional en el salario doméstico. Durante los últimos diez años las restricciones a las empresas mexicanas para participar en los mercados mundiales se han relajado. Como resultado, el uso de insumos importados en el proceso productivo ha aumentado ${ }^{4}$. La apertura de este canal hizo más sensibles a los precios de la industria a movimientos en el tipo de cambio pero menos sensible a variaciones en el salario doméstico. Esto significa que en ausencia de esta apertura comercial, la política de aumentos salariales por encima de la depreciación de la moneda llevada a cabo en México entre 1990 y 1994 se hubiese traducido en un mayor incremento en los precios domésticos medidos en dólares.

Este artículo está organizado de la siguiente manera: la sección 1 presenta la teoría; la sección 2 describe el marco económico en México y los datos utilizados en este estudio; la sección 3 discute las pruebas empíricas llevadas a cabo; la sección 4 presenta los resultados.

\section{Marco Teórico}

3 Incluso si esta función presenta propiedades de elasticidad constante, siempre y cuando existan costos fijos positivos.

${ }^{4}$ Los niveles de deuda en moneda extranjera también han aumentado (ver Conesa (1997)). 
En este apartado se analiza cuidadosamente la relación entre los precios de equilibrio, el tipo de cambio y el salario doméstico, en un modelo donde un bien homogéneo es intercambiado en un mercado oligopólico en el cual las empresas compiten vía cantidades ${ }^{5}$. El modelo de Cournot tradicional es extendido al permitir a las empresas nacionales utilizar insumos importados.

La principal predicción teórica es que la elasticidad tipo de cambio de los precios domésticos medidos en moneda extranjera difiere de la elasticidad salario, no sólo en signo, sino también en magnitud. De igual forma, la suma del pass-through del tipo de cambio y del salario doméstico hacia los precios domésticos medidos en moneda doméstica es menor a uno. Una simple extensión de este resultado implica que los markups de equilibrio pueden cambiar incluso cuando el tipo de cambio y el salario nominal varíen proporcionalmente manteniendo el tipo de cambio real constante. La prueba formal de esto último se presenta en el Apéndice A. También se encontró que el diferencial entre estas dos elasticidades es independiente del uso de insumos importados y que es una función de otros parámetros del modelo.

Para verificar la consistencia de los resultados ante el uso de modelos alternativos de competencia, en el Apéndice B se presenta un modelo alternativo ${ }^{6}$. Este modelo, que es una versión extendida del de Dixit-Stiglitz (DS) (1977), predice que cambios en el tipo de cambio mueven el precio de equilibrio medido en unidades de moneda extranjera, en la dirección exactamente opuesta que cambios en el salario doméstico. La razón de este resultado es que, contrariamente al supuesto de Cournot, la demanda por el bien final en el modelo DS presenta elasticidad constante. Esto implica que el precio de equilibrio es una función lineal del costo unitario de la empresa (e.g. los markups son constantes). Debido a que la función del costo unitario es homogénea de grado uno en el vector de precios de los factores $^{7}$, el pass-through del tipo de cambio y del salario a los precios en moneda doméstica necesariamente suman uno. Sin embargo, si se extiende el marco básico de costos incluyendo un costo fijo, se obtienen todos los resultados principales incluso al mantenerse el supuesto de demanda con elasticidad constante. La razón es que, después de un aumento proporcional en el precio de todos

\footnotetext{
${ }^{5}$ En un modelo tipo Bertrand de competencia vía precios con productos diferenciados se obtienen idénticos resultados.

6 También se analizaron otros dos modelos (los resultados no se presentan aquí) y se encontraron exactamente las mismas conclusiones. Uno de ellos es una extensión del modelo de Salop (1979) de diferenciación parcial, y el otro es un modelo de diferenciación cualitativa basado en Shaked y Sutton (1982).

${ }^{7}$ Independientemente de la tecnología (ver Varian (1992)).
} 
los factores, para alcanzar un markup constante en la presencia de un costo fijo, el precio de equilibrio debe aumentar proporcionalmente más que los precios de los factores.

Se asumió que existen dos tipos de empresas compitiendo en el mercado doméstico: empresas domésticas y extranjeras. Para producir el bien final, las empresas extranjeras utilizan mano de obra y materias primas extranjeras, mientras que las empresas domésticas usan mano de obra nacional y materias primas importadas. Ambos tipos de empresas utilizan una tecnología Leontief ${ }^{8}$ de la forma:

$$
\begin{aligned}
q\left(L, I^{*}\right) & =\min \left\{\frac{L}{1-\gamma}, \frac{I^{*}}{\gamma}\right\} \\
q^{*}\left(L^{*}, I^{*}\right) & =\min \left\{\frac{L^{*}}{1-\gamma}, \frac{I^{*}}{\gamma}\right\}
\end{aligned}
$$

donde $q$ denota la producción de la empresa doméstica, $L$ denota la mano de obra doméstica, e $I$ denota a las materias primas. Los asteriscos representan variables extranjeras.

Para asegurar que no se desperdicia ningún insumo con un precio positivo, la empresa doméstica debe operar en un punto donde $q=\frac{L}{1-\gamma}=\frac{I^{*}}{\gamma}$. Por lo tanto, la empresa deberá utilizar $q(1-\gamma)$ unidades de $L$ y $q \gamma$ unidades de $I^{*}$, independientemente del precio de los insumos. Siguiendo los mismos pasos para la empresa extranjera, encontramos las siguientes funciones de costos totales para empresas domésticas y extranjeras en unidades de moneda doméstica y extranjera respectivamente:

$$
\begin{aligned}
T C\left(w, r^{*}, E, q\right) & =q\left(r^{*} E \gamma+w(1-\gamma)\right) \\
T C *\left(w^{*}, r^{*}, q^{*}\right) & =q^{*}\left(r^{*} \gamma+w^{*}(1-\gamma)\right)
\end{aligned}
$$

donde $T C$ es el costo total de una empresa doméstica medido en unidades de moneda doméstica, $w$ es el costo por unidad de mano de obra doméstica medida en moneda doméstica ${ }^{9}, r^{*}$ es el costo en

${ }^{8}$ Se demostrará que los resultados son consistentes para especificaciones tecnológicas alternativas.

${ }^{9}$ Nótese que el modelo implica que la única fuente de variación en el costo unitario es el salario doméstico. El análisis puede extenderse para incluir un término de productividad sin cambiar los principales resultados. 
moneda extranjera por unidad de materia prima extranjera, y $E$ es el tipo de cambio nominal medido en unidades de moneda doméstica por unidad de moneda extranjera.

Por simplicidad, se asumió que el salario extranjero y el costo de las materias primas extranjeras son constantes e iguales a uno, $w^{*}=r^{*}=1$. Por lo tanto, los costos unitarios de producción de las empresas domésticas y extranjeras medidos en unidades de moneda doméstica y extranjera respectivamente, están dados por:

$$
\begin{gathered}
c(w, E)=E \gamma+w(1-\gamma) \\
c^{*}=1
\end{gathered}
$$

Dado que se asumió que $w^{*}=1$, se puede definir el tipo de cambio real basado en costos unitarios de la mano de obra como TCR $=\frac{E}{w}$. De esta manera, se pueden separar las variaciones en el TCR en variaciones en sus dos componentes:

Intensidad de mano de obra* en la Industria Manufacturera por división

\begin{tabular}{ccccccccc}
\hline & \multicolumn{7}{c}{ División } \\
\cline { 2 - 9 } Year & 1 & 2 & 3 & 4 & 5 & 6 & 7 & 8 \\
1987 & 0.21 & 0.62 & 0.60 & 0.19 & 0.18 & 0.24 & 0.12 & 0.25 \\
1988 & 0.21 & 0.63 & 0.59 & 0.19 & 0.19 & 0.26 & 0.11 & 0.24 \\
1989 & 0.21 & 0.63 & 0.55 & 0.20 & 0.19 & 0.28 & 0.12 & 0.23 \\
1990 & 0.21 & 0.64 & 0.56 & 0.23 & 0.20 & 0.28 & 0.12 & 0.23 \\
1991 & 0.20 & 0.66 & 0.60 & 0.25 & 0.21 & 0.25 & 0.13 & 0.22 \\
1992 & 0.20 & 0.68 & 0.62 & 0.26 & 0.21 & 0.23 & 0.13 & 0.21 \\
1993 & 0.19 & 0.69 & 0.60 & 0.27 & 0.21 & 0.21 & 0.12 & 0.21 \\
1994 & 0.18 & 0.68 & 0.56 & 0.26 & 0.20 & 0.19 & 0.11 & 0.19 \\
1995 & 0.18 & 0.72 & 0.58 & 0.20 & 0.16 & 0.21 & 0.08 & 0.16 \\
\hline
\end{tabular}

* Empleo total entre valor de la producción real.

Fuente: INEGI

Sean $p$ el precio doméstico y $p^{\mathrm{e}}$ es el precio doméstico medido en unidades de moneda extranjera $(p / E)$. Las elasticidades del precio doméstico, medidas en unidades de moneda extranjera, respecto al tipo de cambio nominal ( $\Psi_{E}^{p_{e}}$ ) y al salario doméstico $\left(\Psi_{w}^{p_{e}}\right.$ ) están dadas respectivamente por: 


$$
\begin{gathered}
\Psi_{E}^{p_{e}}=\left.\frac{\hat{p}}{\hat{E}}\right|_{\hat{w}=0}-1=\Psi_{E}^{p}-1 \\
\Psi_{w}^{p_{e}}=\left.\frac{\hat{p}}{\hat{w}}\right|_{\hat{E}=0}=\Psi_{w}^{p}
\end{gathered}
$$

donde $\hat{x}=\frac{d x}{x}$, y $\Psi_{E}^{p}$ representa la elasticidad tipo de cambio del precio doméstico medido en unidades de moneda doméstica.

Se asumió que hay $n$ y $n^{*}$ empresas domésticas y extranjeras respectivamente, compitiendo en el mercado doméstico. La función lineal ${ }^{10}$ de demanda está dada por:

$$
Q=a-b p
$$

donde $Q=n q+n^{*} q^{*}$ es la producción total.

Los ingresos de la empresa doméstica (en unidades de moneda doméstica) y de la empresa extranjera (en unidades de moneda extranjera) están dados respectivamente por las siguientes expresiones:

$$
\begin{aligned}
& \Pi=(p-\gamma E-(1-\gamma) w) q \\
& \Pi^{*}=\left(\frac{p}{E}-1\right) q^{*}
\end{aligned}
$$

La maximización del sistema en (11) nos proporciona el precio de equilibrio y la cantidad producida por la empresa doméstica típica:

$$
p=\frac{a}{b N}+\frac{\left(\gamma \cdot n+n^{*}\right) \cdot E+(1-\gamma) \cdot n \cdot w}{N}
$$

${ }^{10}$ Cambiar de una demanda lineal a una función más general de demanda no altera los principales resultados siempre y cuando la nueva demanda no presente propiedades de elasticidad constantes. Ver Dixit (1986) para la sensibilidad de los precios de equilibrio ante distorsiones en modelos oligopólicos para diferentes funciones de demanda. 


$$
q=\frac{a}{N}+\frac{b}{N} \cdot\left[(w-E)\left(\gamma\left(1+n^{*}\right)-n^{*}\right)-w\right]
$$

donde $N=n+n^{*}+1$.

En un modelo en el que las empresas domésticas utilizan únicamente insumos domésticos, un cambio en el tipo de cambio nominal sólo cambiará la función de reacción de la empresa extranjera. Sin embargo, al usar estos insumos importados tenemos un canal adicional por el cual el tipo de cambio nominal afecta a los precios domésticos.

La gráfica 1.1 muestra la manera en que los cambios en el salario doméstico afectan al equilibrio en este modelo extendido. Las funciones de reacción de la empresa nacional y la empresa extranjera están representadas por las líneas $H H$ y $F F$ respectivamente. El equilibrio inicial está representado por el punto $A$. Si $w$ cae, $H H$ se desplaza hacia arriba sobre la línea $F F$. Este desplazamiento (y el incremento en la cantidad producida por la empresa doméstica) es mayor cuando $\gamma$, que mide la proporción de insumos importados en la producción doméstica, es igual a cero (punto B). En el límite, cuando $\gamma=1$ y el único insumo de producción es importado, el equilibrio no se afectada por variaciones en el salario doméstico.

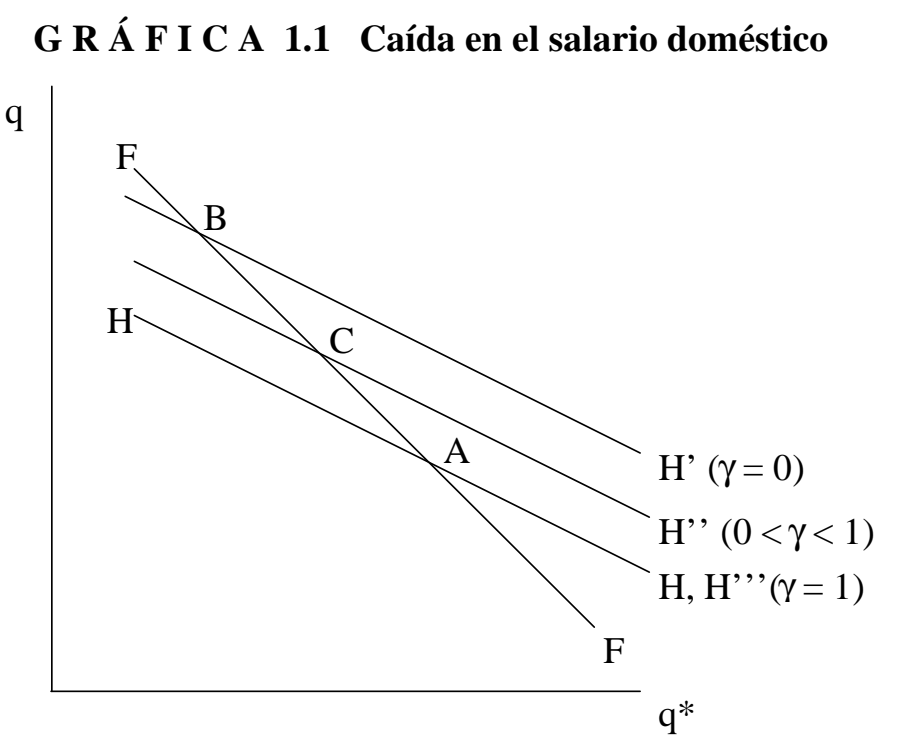

De igual forma, la gráfica 1.2 ilustra la manera en que los cambios en el tipo de cambio nominal alteran el equilibrio en el mercado. Para niveles estrictamente positivos de $\gamma$, un aumento en $E$ desplaza la línea $F F$ a la izquierda y la línea $H H$ hacia abajo. El desplazamiento en $F F$ es independiente de $\gamma$, pero el de $H H$ aumenta con el valor de este parámetro. La intuición es que el costo marginal de la empresa doméstica cambia más cuando $E$ varía si los insumos importados son 
más importantes en el proceso productivo. Cuando $\gamma=1$ las cantidades de equilibrio cambian en la misma proporción, de manera que la razón $\left(q / q^{*}\right)$ permanece constante (punto $D$ ). Cuando $0<\gamma<1$, la cantidad de equilibrio producida por la empresa doméstica puede aumentar o disminuir, dependiendo del número relativo de empresas domésticas y extranjeras compitiendo en el mercado.

\section{G R Á F I C A 1.2 Aumento en el tipo de cambio nominal}

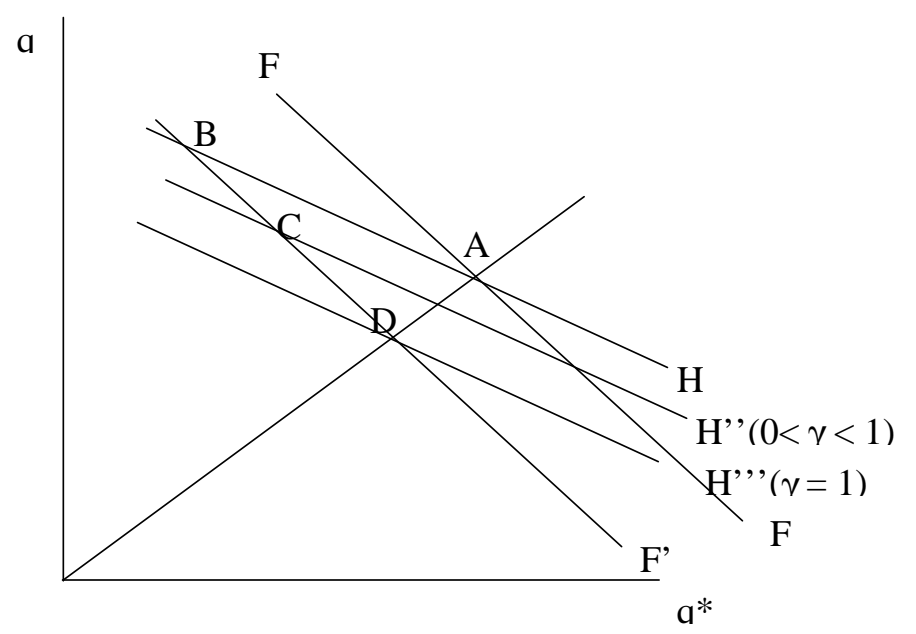

Utilizando (12), se pueden encontrar la elasticidad del precio doméstico, medida en unidades de moneda extranjera, respecto al tipo de cambio y al salario doméstico. Estas elasticidades están dadas respectivamente por:

$$
\begin{aligned}
\Psi_{E}^{p_{e}} & =-\frac{a}{b N p}-\frac{(1-\gamma) w n}{N p} \\
\Psi_{w}^{p_{e}} & =\frac{(1-\gamma) w n}{N p}
\end{aligned}
$$

De manera congruente con los estudios previos, las elasticidades del precio doméstico respecto al tipo de cambio y al salario nominal, son ambas menores a la unidad. Esto significa que una depreciación del tipo de cambio nominal disminuye el precio de equilibrio medido en unidades de moneda extranjera, como lo muestra la ecuación (14). De igual forma, (15) muestra que si el tipo de cambio se mantiene constante, un incremento en el salario doméstico aumenta el precio de equilibrio independientemente de la moneda en que se exprese este último. Este pass-through se reduce a medida que las empresas domésticas utilizan menos mano de obra nacional. 
Combinando (14) y (15), se obtiene el diferencial entre el negativo de la elasticidad tipo de cambio y la elasticidad respecto al salario doméstico:

$$
\Delta \Psi=-\Psi_{E}^{p_{e}}-\Psi_{w}^{p_{e}}=\frac{a}{b N p}
$$

La ecuación (16) tiene dos implicaciones importantes. Primero, si la industria es perfectamente competitiva, movimientos proporcionales en el tipo de cambio y en el salario no afectarán el nivel de precios medido en moneda extranjera $\left(\lim _{N \rightarrow \infty} \Delta \Psi=0\right)$. Segundo, el diferencial entre las dos elasticidades es independiente del uso de insumos importados en el proceso de producción doméstico $(\Delta \Psi$ no es una función de $\gamma$ ).

Utilizando (8) y (9), el diferencial puede ser expresado de manera alternativa como:

$$
\Delta \Psi=\underbrace{1-\frac{d \ln p}{d \ln E}}_{\frac{d \ln p / E}{d \ln E}}-\frac{d \ln p}{d \ln w}
$$

Así, $\Delta \Psi>0$ implica que el pass-through del tipo de cambio y de los salarios domésticos hacia los precios medidos en moneda doméstica son menores a uno incluso combinados (e.g. el pass-through del costo unitario es incompleto). Además, si $\Delta \Psi$ es estrictamente positivo, movimientos en el tipo de cambio real inducidos por cambios en el tipo de cambio nominal tendrán un mayor impacto sobre $p^{\mathrm{e}}$ que movimientos proporcionales en el tipo de cambio real generados por cambios en el salario doméstico. Por ejemplo, si el tipo de cambio se deprecia de tal forma que compense cualquier aumento en el salario, de manera que el tipo de cambio real permanezca constante, los precios en moneda extranjera deberán disminuir. Para ver esto más claramente, sea $\hat{E}=\hat{w}=g>0$, lo que implica que ${ }^{11}$ :

$$
\hat{p}_{e}=\Psi_{E}^{p_{e}} \hat{E}+\Psi_{w}^{p_{e}} \hat{w}=-\Delta \Psi g<0
$$

${ }^{11}$ La ecuación (7) implica que el TCR no se mueve con estos cambios. 
Los pactos concertados en México durante el periodo 1988-1994 invariablemente frenaron la tasa de depreciación con relación al movimiento en los salarios. Como resultado, el TCR se apreció y los precios en dólares aumentaron significativamente (ver siguiente sección). De acuerdo a este modelo, el exceso en el aumento en los salarios sobre el tipo de cambio requerido para mover los precios en moneda extranjera hacia arriba está dado por:

$$
\hat{p}_{e}>0 \Leftrightarrow \frac{\hat{w}}{\hat{E}}>\frac{\Psi_{w}^{p_{e}}+\Delta \Psi}{\Psi_{w}^{p_{e}}}>1
$$

Por lo tanto, en una industria menos competitiva (donde $\Delta \Psi$ es mayor), el aumento en el salario sobre la depreciación de la moneda "requerido" para incrementar $p^{\mathrm{e}}$ es mayor.

Es claro que el análisis presentado hasta el momento se limita a un horizonte de corto plazo. Esto se debe a que no se está incorporando el hecho de que cambios en el tipo de cambio o del salario doméstico afectan no sólo los costos unitarios de las empresas, sino además la riqueza de los consumidores. Esta omisión puede justificarse asumiendo que cambios en estas dos variables toman tiempo para verse reflejados en la demanda de los consumidores por el bien final. Por lo tanto, si se considera un horizonte más amplio, el término independiente de la demanda por el bien final (a) puede ser una función del salario y del tipo de cambio. En este caso, se tiene que si $a=a(w, E)$, entonces (14), (15) y (16) pueden reexpresarse como:

$$
\begin{aligned}
\Psi_{E}^{p_{e}} & =\frac{1}{p}\left[\frac{\partial a}{\partial E} \frac{E}{b N}-\frac{a}{b N}-\frac{(1-\gamma) w n}{N}\right] \\
\Psi_{w}^{p_{e}} & =\frac{1}{p}\left[\frac{\partial a}{\partial w} \frac{w}{b N}+\frac{(1-\gamma) w n}{N}\right] \\
\Delta \Psi & =\frac{1}{b N p}\left[a-\frac{\partial a}{\partial w} w-\frac{\partial a}{\partial E} E\right]
\end{aligned}
$$

Por lo tanto, si la función $a=a(w, E)$ es homogénea de grado uno, en el largo plazo se tiene que $\Delta \Psi=0$. Esto significa que en el largo plazo la elasticidad de $p^{\mathrm{e}}$ respecto al tipo de cambio es igual en magnitud pero de signo opuesto a la elasticidad de $p^{\mathrm{e}}$ respecto al salario doméstico. Sin embargo, el pass-through de cada variable es aún incompleto (e.g. ambas elasticidades son, en valor absoluto, menores a uno). 


\section{Marco Económico en México}

\subsection{Tipo de Cambio, Salarios y Precios Internos.}

Después de que los precios aumentaron en México en más de 150\% durante 1987, se decidió instrumentar un agresivo programa de estabilización heterodoxo que incluía reformas tales como liberalización financiera, la privatización de empresas públicas y la desregulación industrial ${ }^{12}$. En este esfuerzo de estabilización, el tipo de cambio actuó como ancla nominal para romper la inercia inflacionaria ${ }^{13}$. Por medio de una serie de pactos que frenaron la tasa de depreciación de la moneda en relación al movimiento del salario, en 1993 el gobierno logró reducir la inflación a niveles de un solo dígito por primera vez en veinte años ${ }^{14}$. Durante este proceso, el tipo de cambio real (TCR) se apreció de manera constante, generando una pérdida en competitividad y frenando el crecimiento. El esfuerzo de estabilización se vio frenado por el colapso del tipo de cambio en diciembre de $1994^{15}$.

La gráfica 2.1 muestra la forma en que estas políticas se reflejaron en el tipo de cambio real bilateral entre México y Estados Unidos basado en costos unitarios de la mano de obra (TCR). En la gráfica se presentan cambios anuales en el TCR (DTCR), junto con cambios en sus dos componentes: el tipo de cambio nominal $(D E)$ y el salario relativo $(D(w / w *))$. Un valor negativo de DTCR indica una apreciación.

12 Ver Aspe (1993) para un estudio completo de las reformas.

13 Ver Conesa (1997) para los detalles acerca de la política de tipo de cambio nominal seguida durante este periodo.

${ }^{14}$ Ver Dornbush y Werner (1994) para una descripción completa de este proceso.

15 Ver Dombush, Goldfajn y Valdés (1995) para un análisis del tipo de cambio real y el colapso del peso mexicano en 1994. 


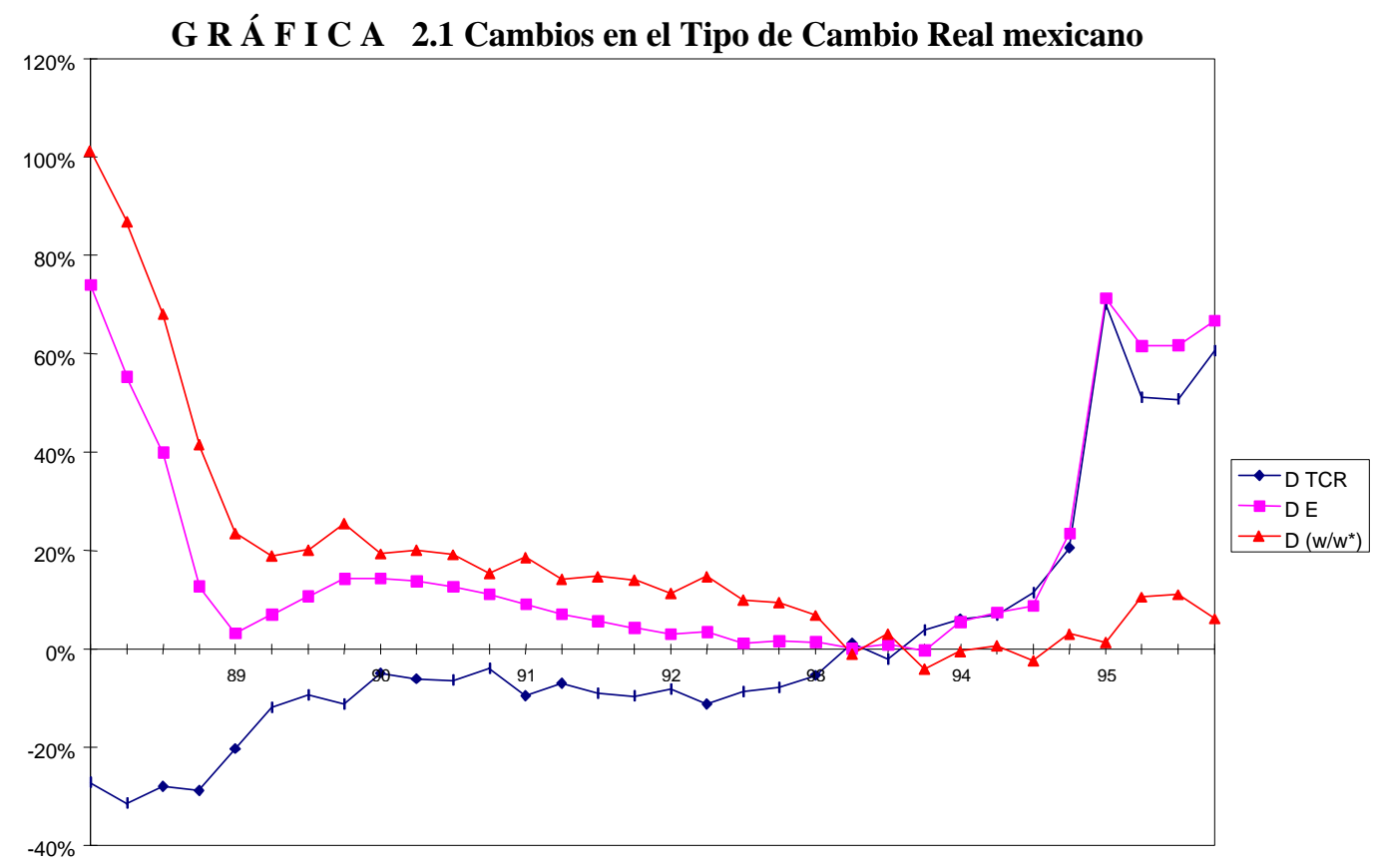

Claramente, el TCR se apreció constantemente desde 1988 hasta 1993. Esto fue resultado de aumentos en el salario relativo muy por encima de la depreciación de la moneda. Sin embargo, a comienzos de 1994 la situación se revirtió. En el último trimestre de 1994, el colapso cambiario llevó a una depreciación significativa del tipo de cambio real. Básicamente, durante el periodo 1988-1993, el TCR fue conducido principalmente por movimientos relativos del salario ${ }^{16}$, mientras que de 1994 a 1995 fue guiado por el tipo de cambio.

Otra consecuencia importante de las políticas cambiarias y salariales descritas anteriormente fue el pronunciado incremento de los precios domésticos medidos en unidades de moneda extranjera. La gráfica 2.2 muestra el logaritmo de la razón del INPC en México y el tipo de cambio bilateral entre México y Estados Unidos ${ }^{17}$.

16 Para mayor precisión, se debería especificar movimientos en el salario doméstico, debido a que la varianza del costo unitario de la mano de obra en Estados Unidos es prácticamente cero.

${ }^{17}$ El comportamiento de los precios en cada sector de la industria manufacturera es bastante similar. Se muestra sólo el INPC para ilustrar el mensaje principal. 
G R Á F I C A 2.2 Índice de precios al consumidor de México en dólares

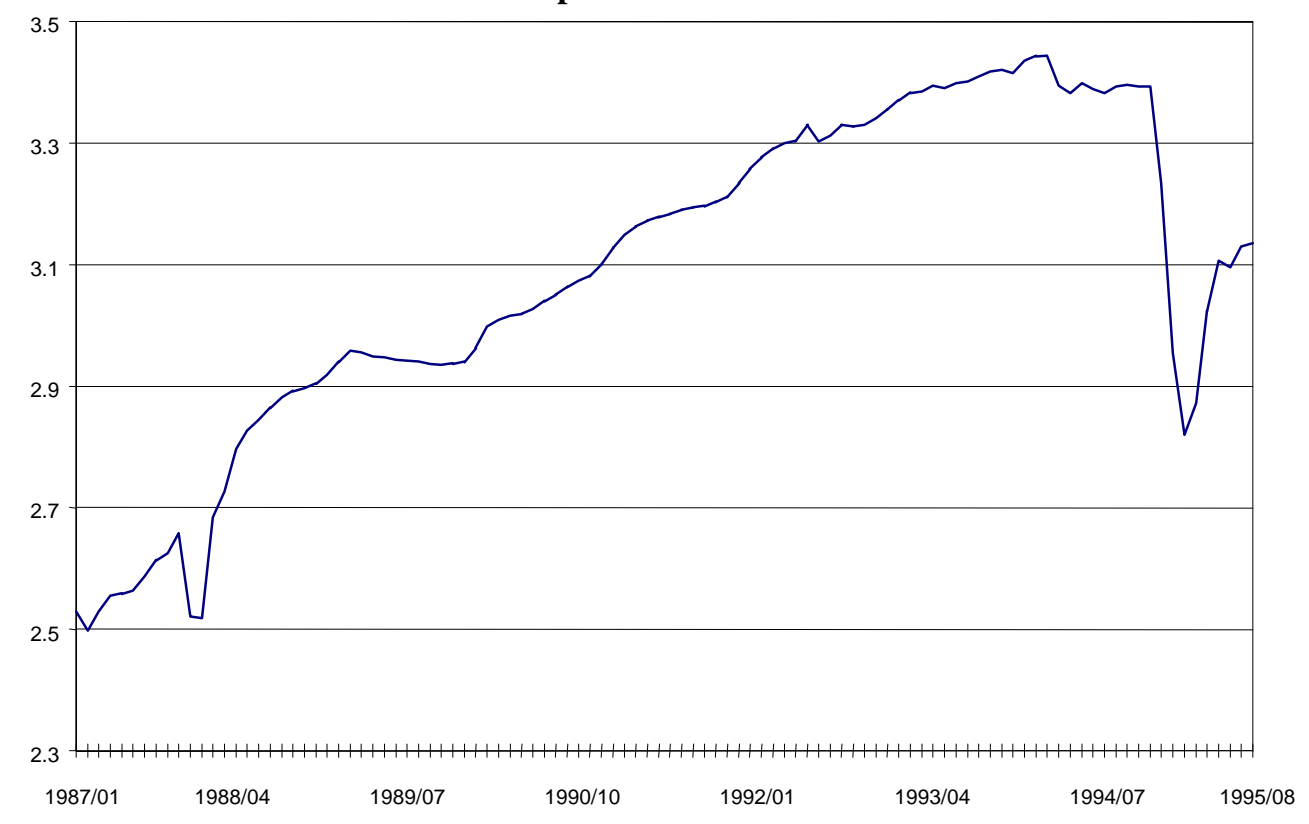

Se observó que los precios domésticos en dólares incrementaron continuamente desde 1988 hasta finales de 1993, periodo en el cual los incrementos en el salario doméstico estuvieron por encima de la depreciación del tipo de cambio. A partir de 1994 esta tendencia se revirtió. Los grandes ajustes cambiarios que comenzaron en diciembre de 1994 redujeron los precios en dólares a niveles alcanzados cinco años atrás.

\subsection{La Industria Manufacturera en México}

Las Cuentas Nacionales en México separan la actividad económica en ocho divisiones. Estas divisiones son a su vez desagregadas en industrias, las cuales son separadas en ramas. La Industria Manufacturera es una de las ocho grandes divisiones y está integrada por ocho subdivisiones y 48 ramas. La definición de cada subdivisión y sus ramas se presenta en el Apéndice C.

México comenzó un profundo proceso de liberalización en 1985, cuando iniciaron las negociaciones para ser incluido en el Acuerdo General Sobre Aranceles y Comercio (GATT). Este proceso de apertura comercial culminó en 1993 con la aprobación formal de un tratado de libre comercio con 
Estados Unidos y Canadá. Estas medidas abrieron nuevos mercados de productos finales e intermedios a las empresas mexicanas ${ }^{18}$.

Una estimación del uso de insumos importados para el periodo 1992-1995 en la Industria Manufacturera en México se presenta en el cuadro siguiente. Para cada una de las 48 ramas, el dato representa la razón del valor de las importaciones realizadas por las 50 empresas más grandes en la rama en relación a la producción total del sector, ajustada por el diferencial de inflación entre México y Estados Unidos ${ }^{19}$.

\section{U A D R O 2.1}

\section{Uso de insumos importados en la Industria Manufacturera en México}

\begin{tabular}{|c|c|c|c|c|c|}
\hline División & Media & Mediana & Desv. Std. & Max & Min \\
\hline Todas & 0.11 & 0.05 & 0.12 & 0.47 & 0.00 \\
\hline 1: Alimentos y bebidas & 0.10 & 0.03 & 0.15 & 0.47 & 0.00 \\
\hline 2: Textiles & 0.03 & 0.03 & 0.02 & 0.05 & 0.00 \\
\hline 3: Madera & 0.03 & 0.03 & 0.02 & 0.04 & 0.01 \\
\hline 4: Papel & 0.11 & 0.11 & 0.12 & 0.20 & 0.01 \\
\hline 5: Química & 0.11 & 0.12 & 0.06 & 0.20 & 0.03 \\
\hline 6: Prods. minerales no metálicos & 0.04 & 0.04 & 0.01 & 0.05 & 0.02 \\
\hline 7: Industrias metálicas & 0.08 & 0.08 & 0.02 & 0.10 & 0.06 \\
\hline 8: Maquinaria y equipo & 0.20 & 0.21 & 0.13 & 0.42 & 0.04 \\
\hline
\end{tabular}

Del cuadro 2.1 se observa que el uso de insumos importados tiene grandes variaciones entre las industrias. La mayoría de los sectores de la división de productos alimenticios, bebidas y tabaco utilizan relativamente pocos insumos importados ${ }^{20}$. En contraste, empresas en la división de sustancias químicas derivadas del petróleo, caucho y plásticos y en la de productos metálicos, maquinaria y equipo dependen relativamente en mayor medida de materias primas extranjeras.

El uso de mano de obra también varía a través de las divisiones. El cuadro 2.2 muestra la razón de la mano de obra total utilizada en relación a la producción real para las ocho divisiones durante el periodo 1987-1995. Las divisiones de textiles, prendas de vestir e industria del cuero y la de industria de la madera y productos de madera (divisiones 2 y 3 respectivamente) son relativamente más

18 Ver G onzález (1996) para un análisis de la manera en que los regímenes comerciales afectan el passthraugh del tipo de cambio.

${ }^{19}$ Los detalles acerca de la construcción de esta variable se describen en el Apéndice C.

20 A excepción de la industria de aceites y grasas vegetales comestibles (sector 17) y la industria de alimentos para animales (sector 18). 
intensivas en mano de obra, mientras que la división de industrias metálicas básicas (división 7) tiene la menor relación de mano de obra a producción.

C U A D R O 2.2

Intensidad de mano de obra* en la Industria Manufacturera por división

\begin{tabular}{ccccccccc}
\hline & \multicolumn{7}{c}{ División } \\
\cline { 2 - 9 } Year & 1 & 2 & 3 & 4 & 5 & 6 & 7 & 8 \\
1987 & 0.21 & 0.62 & 0.60 & 0.19 & 0.18 & 0.24 & 0.12 & 0.25 \\
1988 & 0.21 & 0.63 & 0.59 & 0.19 & 0.19 & 0.26 & 0.11 & 0.24 \\
1989 & 0.21 & 0.63 & 0.55 & 0.20 & 0.19 & 0.28 & 0.12 & 0.23 \\
1990 & 0.21 & 0.64 & 0.56 & 0.23 & 0.20 & 0.28 & 0.12 & 0.23 \\
1991 & 0.20 & 0.66 & 0.60 & 0.25 & 0.21 & 0.25 & 0.13 & 0.22 \\
1992 & 0.20 & 0.68 & 0.62 & 0.26 & 0.21 & 0.23 & 0.13 & 0.21 \\
1993 & 0.19 & 0.69 & 0.60 & 0.27 & 0.21 & 0.21 & 0.12 & 0.21 \\
1994 & 0.18 & 0.68 & 0.56 & 0.26 & 0.20 & 0.19 & 0.11 & 0.19 \\
1995 & 0.18 & 0.72 & 0.58 & 0.20 & 0.16 & 0.21 & 0.08 & 0.16 \\
\hline
\end{tabular}

* Empleo total entre valor de la producción real.

Fuente: INEGI

El siguiente cuadro muestra estadísticas descriptivas de la razón de concentración para cada división del sector manufacturero. El mayor cociente es alcanzado en la división 7, la cual incluye las industrias básicas de hierro y acero así como a las industrias básicas de metales no ferrosos. Las divisiones 3 y 4, que incluyen sectores como el de la producción de corcho, industrias de imprentas y editoriales, son las más competitivas.

\section{U A D R O 2.3}

Razones de concentración* en la Industria Manufacturera en México

\begin{tabular}{lccccc}
\hline \multicolumn{1}{c}{ División } & Media & Mediana & Desv. Std. & Max & Min \\
Todas & 0.51 & 0.49 & 0.17 & 0.83 & 0.23 \\
1: Alimentos y bebidas & 0.41 & 0.32 & 0.20 & 0.82 & 0.23 \\
2: Textiles & 0.51 & 0.47 & 0.10 & 0.64 & 0.41 \\
3: Madera & 0.39 & 0.39 & 0.01 & 0.40 & 0.39 \\
4: Papel & 0.38 & 0.38 & 0.04 & 0.41 & 0.36 \\
5: Química & 0.53 & 0.52 & 0.13 & 0.75 & 0.26 \\
6: Prods. minerales no metálicos & 0.58 & 0.69 & 0.23 & 0.73 & 0.31 \\
7: Industrias metálicas & 0.80 & 0.80 & 0.04 & 0.83 & 0.78 \\
8: Maquinaria y equipo & 0.58 & 0.57 & 0.11 & 0.79 & 0.43 \\
\hline
\end{tabular}

Promedio ponderado de la razón de concentración de las tres mayores empresas de cada división del sector manufacturero.

Datos del censo manufacturero, INEGI 1993 


\section{Estrategia Empírica}

Para el estudio empírico se utilizaron series de datos mensuales de precio, salario y tipo de cambio nominal desde enero de 1987 a agosto de 1995 inclusive $^{21}$. Se excluyó del análisis al sector automotriz (rama 56) debido al tratamiento particular de importaciones ${ }^{22}$.

Una convención en los estudios de series de tiempo es probar, en primer lugar, la estacionareidad de las variables. La razón de esta medida es que, si las series muestran propiedades no estacionarias (e.g. tienen raíz unitaria), los estadísticos de las regresiones de mínimos cuadrados ordinarios (MCO) no mantendrían sus distribuciones tradicionales, y los estimadores podrían estar sesgados. Si se encuentra que los datos tienen raíces unitarias, una correcta diferenciación resolvería el problema de no estacionareidad con el costo de perder la información de largo plazo de las series. Sin embargo, si se puede encontrar una relación de cointegración, debe ser explotada llevando a cabo una estimación en el marco de un modelo de corrección de errores (MCE) $)^{23}$. La primera etapa de este procedimiento proporciona una estimación de las elasticidades de largo plazo, mientras que en la segunda etapa se pueden encontrar las relaciones de corto plazo, además de arrojar una prueba adicional de cointegración.

\subsection{Pruebas de Estacionareidad y Cointegración}

El cuadro 3.1 muestra los resultados de las pruebas de estacionareidad de Dickey-Fuller aplicadas a las 56 variables $^{24}$ en la muestra. Se puede rechazar la hipótesis nula de estacionareidad a un nivel de $5 \%$ en 53 casos. Puede demostrarse que la hipótesis nula de estacionareidad no puede rechazarse con las variables expresadas en primeras diferencias (i.e., las variables presentan integración de primer orden). En el cuadro 3.1 también se presentan los resultados de las pruebas aumentadas de

${ }^{21}$ Cabe mencionar que cada ejercicio empírico se realizó también excluyendo 1995 y se encontraron los mismos resultados. De esta manera, se verificó que los resultados no se debieron simplemente a los importantes ajustes del tipo de cambio nominal ocurridos durante este año.

22 Las empresas en este sector se encontraron sujetas a restricciones de importaciones basadas en sus exportaciones.

23 Ver D wyer, Kent y Pease (1994) para otra aplicación de MCE al análisis del passthraugh

${ }^{24}$ Cuarenta y siete precios, ocho variables de salario y el tipo de cambio. 
cointegración de Dickey-Fuller ${ }^{25}$. En 36 de 47 ramas no se puede rechazar la hipótesis de estacionareidad de los residuales, lo que indica que en estas ramas las variables están cointegradas.

C U A D RO 3.1

Pruebas de estacionalidad y cointegración

\begin{tabular}{|c|c|c|}
\hline & Estacionalidad & Cointegración \\
\hline & \multicolumn{2}{|c|}{ Valores críticos } \\
\hline $1 \%$ & -3.503 & -4.438 \\
\hline $5 \%$ & -2.893 & -3.826 \\
\hline $10 \%$ & -2.583 & -3.513 \\
\hline Variable * & \multicolumn{2}{|c|}{ Estadístico Dickey-Fuller } \\
\hline $\mathrm{p}^{\mathrm{e}}{ }_{11}$ & -1.434 & -2.980 \\
\hline $\mathrm{p}^{\mathrm{e}}{ }_{12}$ & -1.558 & -3.894 \\
\hline $\mathrm{p}^{\mathrm{e}}{ }_{13}$ & -1.272 & -4.352 \\
\hline $\mathrm{p}_{14}^{\mathrm{e}}$ & -1.481 & -2.885 \\
\hline $\mathrm{p}^{\mathrm{e}}{ }_{15}$ & -1.493 & -3.129 \\
\hline$p^{e}{ }_{16}$ & -1.533 & -4.054 \\
\hline $\mathrm{p}^{\mathrm{e}}{ }_{17}$ & -1.136 & -4.900 \\
\hline $\mathrm{p}^{\mathrm{e}}{ }_{18}$ & -2.517 & -4.496 \\
\hline $\mathrm{p}^{\mathrm{e}}{ }_{19}$ & -1.421 & -4.475 \\
\hline $\mathrm{p}_{20} \mathrm{e}^{\mathrm{s}}$ & -1.227 & -4.005 \\
\hline $\mathrm{p}_{21}^{\mathrm{e}}$ & -1.801 & -5.104 \\
\hline $\mathrm{p}_{22}$ & -1.066 & -4.075 \\
\hline$p^{e}{ }^{2}$ & -0.695 & -1.826 \\
\hline$p_{24}^{e}$ & -2.038 & -4.730 \\
\hline$p_{25}^{e}$ & -1.252 & -3.309 \\
\hline$p^{e} 26$ & -1.606 & -4.645 \\
\hline $\mathrm{p}^{\mathrm{e}} 27$ & -1.352 & -4.864 \\
\hline $\mathrm{p}_{28}^{\mathrm{e}}$ & -1.916 & -3.712 \\
\hline $\mathrm{p}_{2.9}$ & -0.847 & -3.147 \\
\hline $\mathrm{p}_{30}^{\mathrm{e}}$ & -1.594 & -4.631 \\
\hline$p_{31}^{e}$ & -2.280 & -4.597 \\
\hline $\mathrm{p}^{\mathrm{e}} 32$ & -1.169 & -2.839 \\
\hline $\mathrm{p}_{33}^{\mathrm{e}}$ & -1.425 & -4.496 \\
\hline $\mathrm{p}_{34}$ & 0.854 & -4.860 \\
\hline $\mathrm{p}_{35}$ & -1.403 & -4.398 \\
\hline $\mathrm{p}_{36}^{e^{30}}$ & -1.578 & -4.597 \\
\hline $\mathrm{p}^{\mathrm{e}}{ }_{37}$ & -1.544 & -4.057 \\
\hline $\mathrm{p}_{38}^{\mathrm{e}}$ & -1.217 & -3.084 \\
\hline $\mathrm{p}_{3.9}^{\mathrm{e}}$ & -1.786 & -4.915 \\
\hline $\mathrm{p}_{40}^{\mathrm{e}}$ & -1.840 & -5.958 \\
\hline $\mathrm{p}^{\mathrm{e}}{ }_{41}$ & -1.773 & -5.352 \\
\hline $\mathrm{p}_{42}$ & -2.707 & -4.492 \\
\hline $\mathrm{p}_{43}$ & -1.988 & -3.347 \\
\hline $\mathrm{p}_{44}^{\mathrm{e}}$ & -1.199 & -2.733 \\
\hline $\mathrm{p}_{45}$ & -1.134 & -4.116 \\
\hline $\mathrm{p}_{46}^{\mathrm{e}}$ & -2.692 & -4.058 \\
\hline $\mathrm{p}_{47}{ }_{40}$ & -1.958 & -4.314 \\
\hline $\mathrm{p}_{48}^{\mathrm{e}}$ & -1.634 & -3.441 \\
\hline $\mathrm{p}_{49}^{\mathrm{e}}$ & -1.926 & -3.748 \\
\hline $\mathrm{p}_{50}$ & -2.704 & -3.742 \\
\hline $\mathrm{p}_{51}$ & -1.765 & -4.737 \\
\hline $\mathrm{p}_{52}$ & -2.596 & -3.300 \\
\hline$p_{53}$ & -2.085 & -3.692 \\
\hline $\mathrm{p}_{54}$ & -2.620 & -3.852 \\
\hline $\mathrm{p}_{55}$ & -2.304 & -5.210 \\
\hline$p^{e} 57$ & -1.205 & -3.625 \\
\hline $\mathrm{p}_{58}^{\mathrm{e}}$ & -2.736 & -3.592 \\
\hline $\mathrm{EXCH}$ & 0.542 & \\
\hline W1 & -2.100 & \\
\hline W2 & -3.528 & \\
\hline W3 & -2.168 & \\
\hline W4 & -2.589 & \\
\hline W5 & -2.893 & \\
\hline W6 & -1.860 & \\
\hline W7 & -3.064 & \\
\hline W8 & -2.358 & \\
\hline
\end{tabular}

* En logaritmos naturales

$p^{e}{ }_{i}$ es the precio en dólares $(p / E)$ en el sector $i=11 \ldots 58$.

${ }^{25}$ La relación de cointegración tiene al precio en moneda extranjera en el lado izquierdo y al tipo de cambio y al salario en el lado derecho. 
Debe mencionarse que la fuerza de estas pruebas de cointegración ha sido tema de debate por un largo tiempo. Kremers et al. (1992) demuestran que una prueba de significancia en el término de corrección de errores del MCE es de hecho más poderosa que la prueba anterior basada en los residuales de la relación de cointegración. Estas pruebas de significancia de los términos de corrección de errores muestran que la hipótesis nula de no cointegración se rechaza en 40 de 47 sectores $^{26}$.

\subsection{Representación del Modelo de Corrección de Errores}

El primer paso de este procedimiento ${ }^{27}$ consiste en correr la regresión que implica la teoría con los regresores expresados en niveles. Esto provee una estimación de la relación de largo plazo del pass-through de los precios en cada sector:

$$
\mathrm{p}_{i t}^{e}=\phi_{0}+\phi_{1} \mathrm{e}_{t}+\phi_{2} \mathrm{w}_{i t}+\sum_{j=3}^{14} \phi_{j} d u m_{j}+\varepsilon_{i t}
$$

donde $\mathrm{p}^{\mathrm{e}}$ es el logaritmo natural del precio medido en dólares, e es el logaritmo natural del tipo de cambio, w es el logaritmo natural del salario nominal en pesos, dum es una variable dummy mensual para corregir estacionalidad, y $\varepsilon_{i t}$ es el error de largo plazo. En este marco, $\hat{\phi}_{1}$ y $\hat{\phi}_{2}$ representan las elasticidades tipo de cambio y salario de largo plazo respectivamente. Los residuales ajustados, $\hat{\varepsilon}_{i t}$ son entonces sustituidos en una regresión en diferencias para llegar a la representación de la corrección de errores. Los residuales son denominados "término de corrección de errores" y pueden ser interpretados como el reflejo de respuestas a desequilibrios. Formalmente, en el caso de dos rezagos $^{28}$, la ecuación (20) puede ser re-expresada como:

$$
\Delta \mathrm{p}_{i t}^{e}=\delta+\pi_{1} \Delta \mathrm{p}_{i t-1}^{e}+\beta_{1} \Delta \mathrm{e}_{t}+\beta_{2} \Delta \mathrm{e}_{t-1}+\lambda_{1} \Delta \mathrm{w}_{i t}+\lambda_{2} \Delta \mathrm{w}_{i t-1}+\alpha_{1} \hat{\varepsilon_{t-1}}+\sum_{j=1}^{11} \tilde{\phi_{j}} d u m_{j}+v_{t}
$$

\footnotetext{
${ }^{26}$ Los resultados de estas pruebas se encuentran observando el estadístico t de $\alpha_{1}$ en los resultados del MCE descritos en las tablas siguientes.

27 Ver Hamilton (1994) para una descripción completa de modelos de corrección de errores.

${ }^{28}$ Se probó la especificación de diferencia hasta con cuatro rezagos, encontrando que, para el salario doméstico, el tercer y cuarto rezago no eran significativos.
} 
donde $\Delta x_{\mathrm{t}}=x_{\mathrm{t}}-x_{\mathrm{t}-1}$, $\mathrm{y} \vee$ es el error de corto plazo. Las elasticidades de corto plazo respecto al tipo de cambio nominal y al salario nominal están dadas por $\left(\hat{\beta}_{1}+\hat{\beta}_{2}\right)$ y $\left(\hat{\lambda}_{1}+\hat{\lambda}_{2}\right)^{\mathbf{2 9}}$, respectivamente. Nótese que la especificación (21) involucra mezclar datos en niveles (el término de corrección de errores) con datos en diferencias en la misma ecuación. Sin embargo, las variables en primeras diferencias y el término de corrección de errores son estacionarios, lo que a su vez se traduce en estimaciones eficientes de los coeficientes de la especificación (21). Además, como se mencionó anteriormente, una prueba del coeficiente del término de corrección de errores $(\hat{\alpha})$ proporciona una prueba más poderosa de cointegración ${ }^{30}$ que el procedimiento tradicional de Dickey-Fuller. En ambas etapas del MCE utilizamos MCO.

Utilizando los coeficientes estimados para la especificación de corto plazo ((21)), podemos probar $\Delta \Psi=0$. Esto se lleva a cabo mediante una prueba $F$ de restricciones lineales donde la hipótesis nula $\mathrm{es}^{31}$ :

$$
H_{0}: \hat{\beta}_{1}+\hat{\beta}_{2}+\hat{\lambda}_{1}+\hat{\lambda}_{2}=0
$$

Finalmente, la teoría predice que $\Delta \Psi$ debe incrementarse con la concentración de la industria y ser independiente de la proporción de insumos importados. Para probar estas predicciones se estimó la siguiente regresión:

$$
\Delta \Psi_{i}=\beta_{i}^{E}-\lambda_{i}^{w}=\theta_{1} C_{i}+\theta_{2} \gamma_{i}+\eta
$$

donde $\beta^{\mathrm{E}}=-\left(\hat{\beta}_{1}+\hat{\beta}_{2}\right)$ y $\lambda^{\mathrm{w}}=\hat{\lambda}_{1}+\hat{\lambda}_{2}, C$ es la razón de concentración de la industria, $\gamma$ es la estimación de insumos importados, y $\eta$ es un error. Por lo tanto, $\theta_{1}$ deberá ser significativamente positivo y $\theta_{2}$ no debería ser significativamente distinto de cero.

${ }^{29}$ Estrictamente hablando, las elasticidades de corto plazo correctas deberían de calcularse usando una función de impulso-respuesta (para tomar en cuenta el cambio rezagado en precios y el término de corrección de errores).

$30 \mathrm{Si}$ las series están de hecho cointegradas, entonces el coeficiente estimado debería ser negativo y tener un valor absoluto menor a uno.

31 Ver la ecuación (16) 
El último ejercicio analiza la relación entre las elasticidades (de largo plazo) de las elasticidades tipo de cambio y salario del precio medido en moneda doméstica ${ }^{32}$. Utilizando (12), se espera que el pass-through del tipo de cambio a los precios domésticos aumente con $\gamma$, y el passthrough del salario disminuya con este parámetro.

\section{Resultados}

La gráfica 4.1 muestra las elasticidades de largo plazo estimadas respecto al tipo de cambio y al salario de la especificación (20) para los 47 sectores $\left(\hat{\phi_{1}}\right.$ y $\hat{\phi_{2}}$ respectivamente). La mayoría de las observaciones se encuentran cerca de la línea de $45^{\circ}$, lo que significa que no existe diferencia significativa entre las dos estimaciones de largo plazo (e.g. $\Delta \Psi$ en el largo plazo es cercano a cero).

\section{G R Á F I C A 4.1 Elasticidades estimadas de largo plazo}

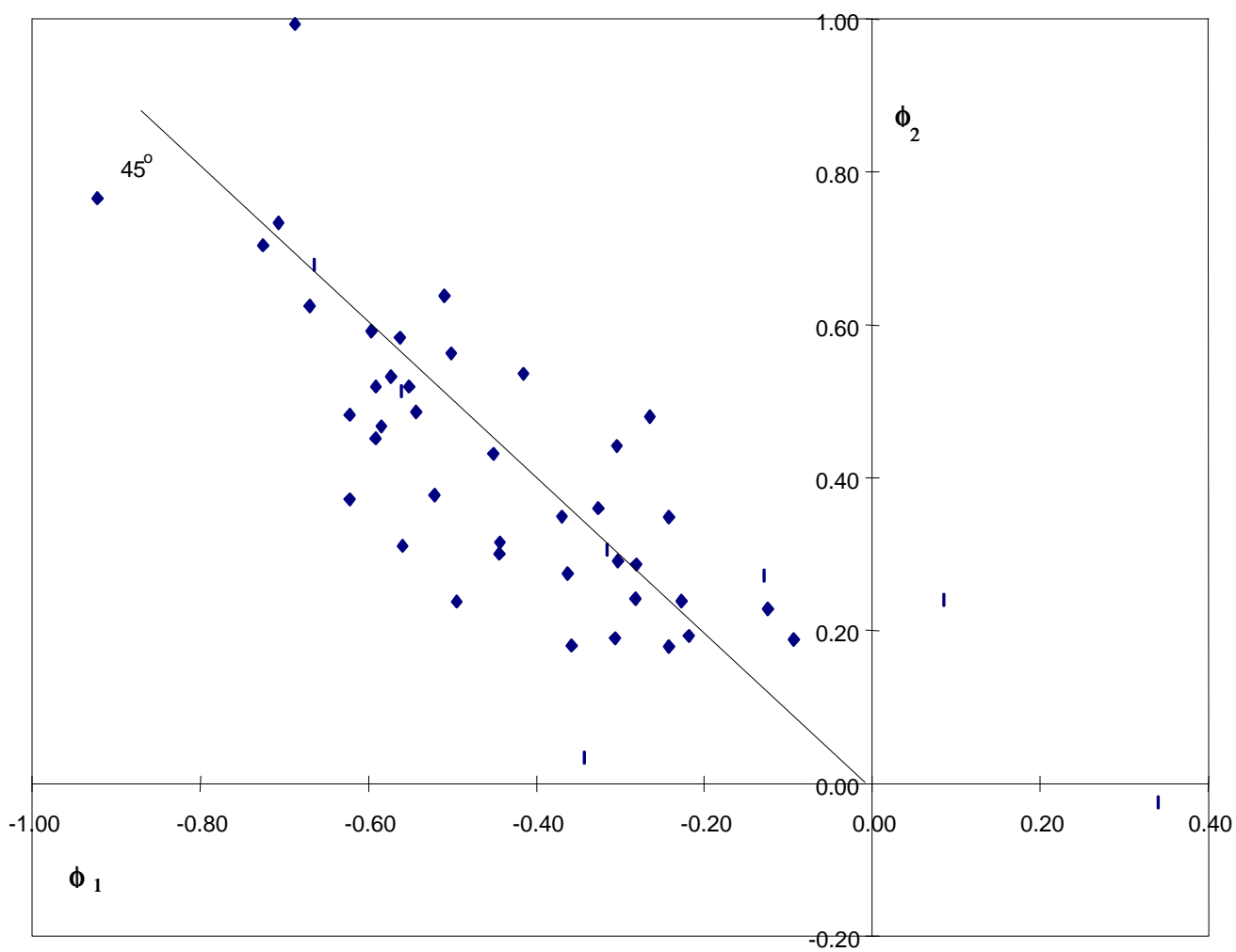

32 Estas elasticidades se obtienen de estimar la especificación (21), pero usando el logaritmo natural de los precios domésticos en pesos en el lado izquierdo. 
La dinámica de corto plazo muestra un cuadro totalmente distinto. En el siguiente grupo de cuadros se muestran los resultados de la especificación (21) para las distintas divisiones de la Industria Manufacturera.

\subsection{Pass-through para la división 1}

Como se puede observar en el cuadro 4.1, la elasticidad tipo de cambio tiene el signo correcto en cada sector (e.g. $\beta_{1}+\beta_{2}<0$ ), y la elasticidad salario es positiva en 12 de 13 sectores. Además, en 9 de los sectores en esta división podemos rechazar estadísticamente la hipótesis $\Delta \Psi=0$. Una simple inspección muestra que en cada caso el negativo de la elasticidad tipo de cambio es mayor que la elasticidad salario (e.g. $\Delta \Psi>0$ ). 


\section{U A D R O 4.1}

Elasticidades estimadas de corto plazo para industria de productos alimenticios, bebidas y tabaco

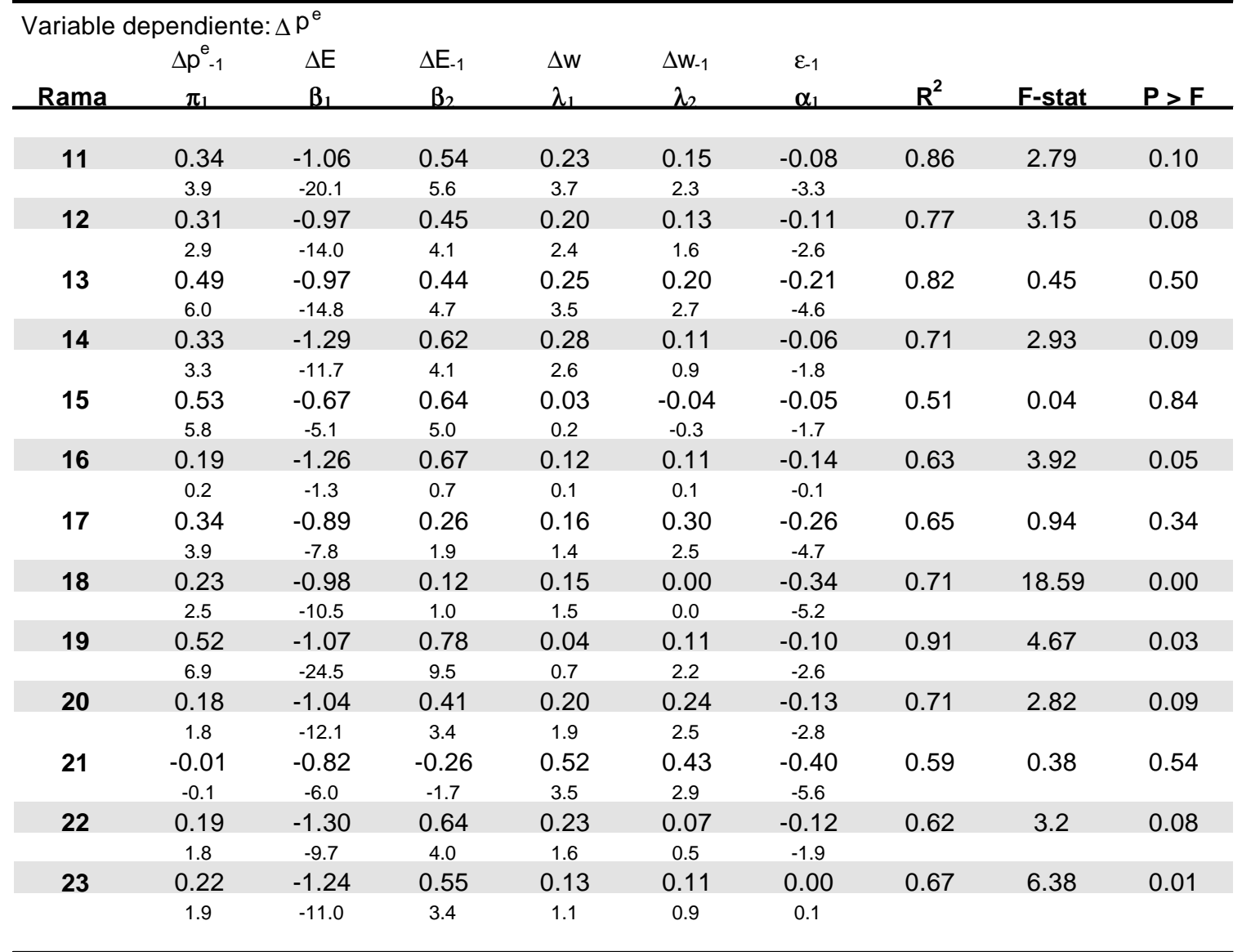

El número debajo de cada coeficiente representa su estadístico t. El número de observaciones es 102. El estadístico $F$ corresponde a la prueba $H_{0}=\beta_{1}+\beta_{2}+\lambda_{1}+\lambda_{2}=0$. Las regiones sombreadas muestran las ramas para las cuales se rechaza esta hipótesis a un nivel del $10 \%$ o meior.

\subsection{Pass-through para las divisiones 2,3 y 4}

El cuadro 4.2 muestra los resultados combinados para las divisiones 2, 3 y 4. Las elasticidades tipo de cambio y salario tienen los signos correctos en cada caso. Del mismo modo que para la división 1, una simple inspección confirma que $\Delta \Psi>0$ para cada rama, y para la rama de textiles, prendas de vestir e industria del cuero, se puede rechazar estadísticamente la hipótesis nula de $\Delta \Psi=0$. 
C U A D R O 4.2

Elasticidades estimadas de corto plazo para las divisiones 2,3 y 4

\begin{tabular}{|c|c|c|c|c|c|c|c|c|c|}
\hline \multicolumn{10}{|c|}{ Variable dependiente: $\Delta \mathrm{p}^{\mathrm{e}}$} \\
\hline & $\Delta \mathrm{p}_{-1}^{\mathrm{e}}$ & $\Delta \mathrm{E}$ & $\Delta \mathrm{E}_{-1}$ & $\Delta \mathrm{W}$ & $\Delta \mathrm{W}_{-1}$ & $\varepsilon_{-1}$ & & & \\
\hline Rama & $\pi_{1}$ & $\beta_{1}$ & $B_{2}$ & $\lambda_{1}$ & $\lambda_{2}$ & $\alpha_{1}$ & $\mathbf{R}^{2}$ & F-stat & $P>F$ \\
\hline \multicolumn{10}{|c|}{ División 2: Textiles, prendas de vestir e industria del cuero } \\
\hline \multirow[t]{2}{*}{24} & 0.32 & -0.73 & 0.33 & 0.08 & 0.05 & -0.23 & 0.65 & 7.04 & 0.01 \\
\hline & 3.6 & -8.9 & 3.1 & 1.6 & 1.1 & -3.8 & & & \\
\hline \multirow[t]{2}{*}{25} & -0.01 & -1.39 & 0.48 & 0.25 & 0.05 & -0.14 & 0.44 & 5.76 & 0.02 \\
\hline & -0.1 & -6.7 & 2.1 & 2.0 & 0.4 & -2.5 & & & \\
\hline \multirow[t]{2}{*}{26} & 0.22 & -1.00 & 0.48 & 0.19 & 0.11 & -0.17 & 0.65 & 3.27 & 0.07 \\
\hline & 2.3 & -9.9 & 3.7 & 2.9 & 1.8 & -3.0 & & & \\
\hline \multirow[t]{2}{*}{27} & 0.47 & -0.99 & 0.56 & 0.15 & 0.05 & -0.12 & 0.83 & 8.71 & 0.00 \\
\hline & 5.5 & -16.4 & 5.7 & 4.0 & 1.2 & -2.8 & & & \\
\hline \multirow[t]{2}{*}{28} & 0.38 & -1.13 & 0.67 & 0.16 & 0.07 & -0.07 & 0.78 & 5.82 & 0.02 \\
\hline & 4.2 & -14.8 & 5.9 & 3.3 & 1.4 & -2.1 & & & \\
\hline \multicolumn{10}{|c|}{ División 3: Industria de la madera y productos de madera } \\
\hline \multirow[t]{2}{*}{29} & 0.20 & -1.15 & 0.33 & 0.40 & 0.27 & -0.11 & 0.68 & 1.21 & 0.27 \\
\hline & 2.1 & -11.0 & 2.2 & 4.3 & 2.8 & -2.9 & & & \\
\hline \multirow[t]{2}{*}{30} & 0.35 & -1.01 & 0.46 & 0.23 & 0.06 & -0.17 & 0.77 & 6.40 & 0.01 \\
\hline & 3.6 & -13.5 & 3.7 & 3.5 & 0.9 & -3.4 & & & \\
\hline \multicolumn{10}{|c|}{ División 4: Papel, productos de papel, imprentas y editoriales } \\
\hline \multirow[t]{2}{*}{31} & 0.54 & -1.02 & 0.81 & 0.11 & 0.02 & -0.08 & 0.87 & 1.18 & 0.28 \\
\hline & 8.6 & -17.4 & 9.9 & 2.7 & 0.5 & -2.7 & & & \\
\hline \multirow[t]{2}{*}{32} & 0.49 & -0.91 & 0.66 & 0.05 & 0.02 & -0.03 & 0.76 & 5.38 & 0.02 \\
\hline & 5.1 & -13.5 & 7.0 & 1.0 & 0.4 & -0.9 & & & \\
\hline
\end{tabular}

Ver notas del cuadro 4.1

\subsection{Pass-through para la división 5}

Como puede observarse en el cuadro 4.3, excepto en dos casos, no se puede rechazar la hipótesis de que las elasticidades tipo de cambio y salario son distintas en la división 5. Además, las ramas 33 (refinación de petróleo), 34 (petroquímica básica), 36 (abonos y fertilizantes), y 40 (otras industrias químicas) son los únicos sectores de la muestra donde la elasticidad salario de los precios en dólares es mayor que (el negativo de) la elasticidad tipo de cambio $\left(\lambda_{1}+\lambda_{2}-\beta_{1}-\beta_{2}>0\right)$, lo que implica que, para estos sectores, $\Delta \Psi<0$. 


\section{U A D R O 4.3}

Elasticidades estimadas de corto plazo para la industria de sustancias químicas

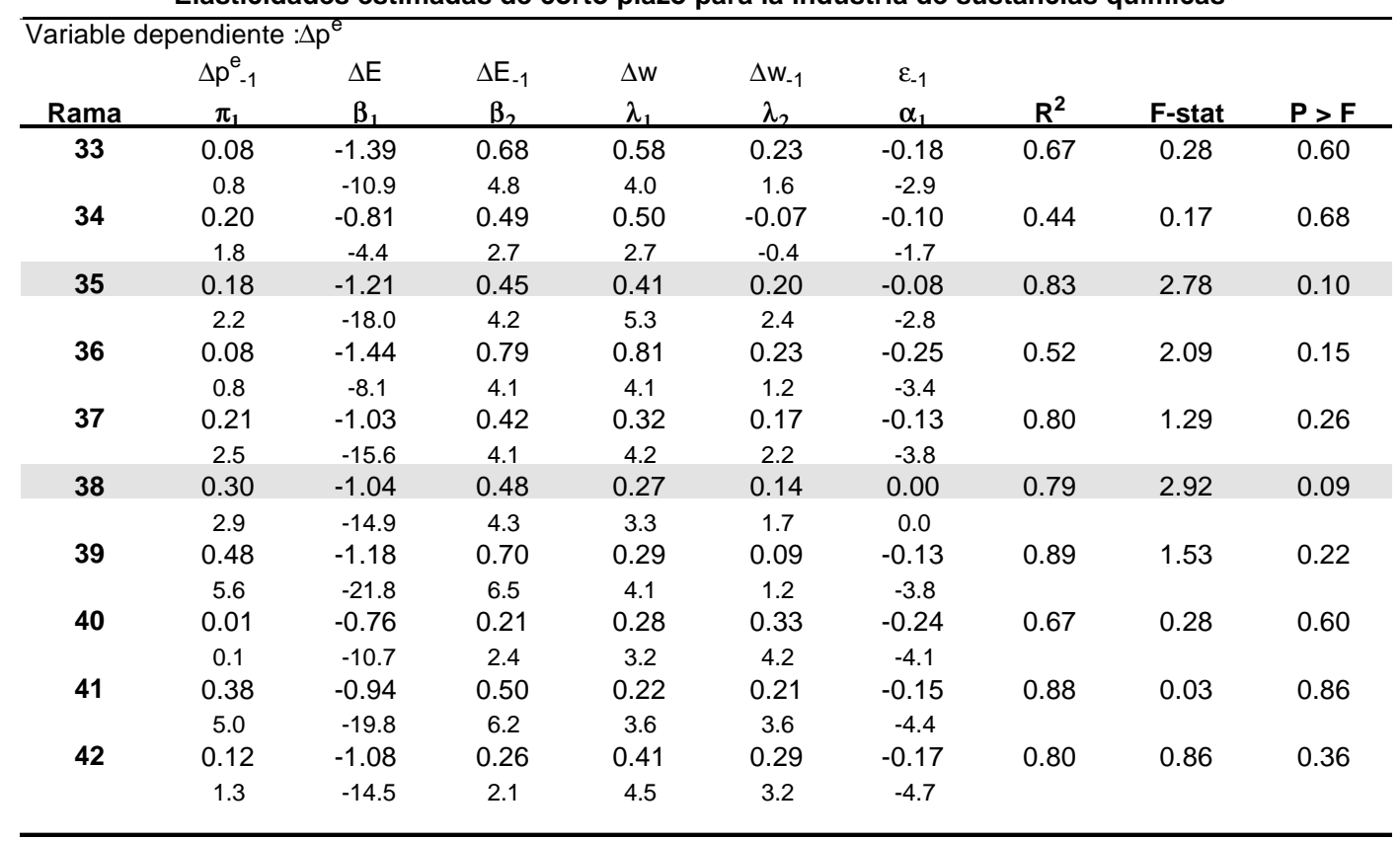

Ver notas del cuadro 4.1

\subsection{Pass-through para las divisiones 6, 7 y 8.}

En los 15 sectores de estas tres divisiones, (el negativo de) la elasticidad tipo de cambio es mayor que la elasticidad salario, a juzgar por los resultados del cuadro 4.4. La diferencia es estadísticamente significativa en nueve casos. Ambos sectores de la división 7 muestran una elasticidad salario relativamente baja, un resultado consistente con que este sector es el menos intensivo en mano de obra en la Industria Manufacturera. 


\section{U A D R O 4.4}

Elasticidades estimadas de corto plazo para las divisiones 6,7 y 8

\begin{tabular}{|c|c|c|c|c|c|c|c|c|c|}
\hline \multicolumn{10}{|c|}{ Variable dependiente: $\Delta \mathrm{p}^{\mathrm{e}}$} \\
\hline \multirow{3}{*}{ Rama } & $\Delta p_{-1}^{e}$ & $\Delta \mathrm{E}$ & $\Delta \mathrm{E}_{-1}$ & $\Delta \mathrm{w}$ & $\Delta \mathrm{W}_{-1}$ & $\varepsilon_{-1}$ & & & \\
\hline & $\pi_{1}$ & $\beta_{1}$ & $\mathrm{~B}_{2}$ & $\lambda_{1}$ & $\lambda_{2}$ & $\alpha_{1}$ & $\mathbf{R}^{2}$ & F-stat & $P>F$ \\
\hline & \multicolumn{9}{|c|}{ División 6: Productos de minerales no metálicos excepto petróleo y carbón } \\
\hline \multirow[t]{2}{*}{43} & 0.35 & -1.11 & 0.59 & 0.12 & 0.12 & -0.08 & 0.85 & 9.18 & 0.00 \\
\hline & 4.3 & -17.7 & 5.0 & 2.1 & 2.2 & -2.7 & & & \\
\hline \multirow[t]{2}{*}{44} & 0.15 & -0.83 & 0.38 & 0.21 & 0.09 & -0.06 & 0.58 & 1.48 & 0.23 \\
\hline & 1.4 & -8.0 & 2.9 & 2.4 & 1.0 & -1.3 & & & \\
\hline \multirow[t]{2}{*}{45} & 0.56 & -1.02 & 0.64 & 0.19 & 0.04 & -0.10 & 0.87 & 3.76 & 0.06 \\
\hline & 6.6 & -17.8 & 5.6 & 3.6 & 0.7 & -3.1 & & & \\
\hline \multicolumn{10}{|c|}{ División 7: Industrias metálicas básicas } \\
\hline \multirow[t]{2}{*}{46} & 0.43 & -1.03 & 0.50 & 0.13 & 0.02 & -0.22 & 0.70 & 10.85 & 0.00 \\
\hline & 5.0 & -10.5 & 3.8 & 2.7 & 0.5 & -4.2 & & & \\
\hline \multirow{2}{*}{47} & 0.43 & -0.60 & 0.49 & 0.05 & 0.01 & -0.16 & 0.57 & 0.22 & 0.64 \\
\hline & 5.0 & -6.4 & 4.8 & 1.1 & 0.2 & -3.3 & & & \\
\hline \multicolumn{10}{|c|}{ División 8: Productos metálicos, maquinaria y equipo } \\
\hline \multirow[t]{2}{*}{48} & 0.38 & -1.00 & 0.60 & 0.18 & 0.12 & -0.11 & 0.74 & 1.01 & 0.32 \\
\hline & 4.2 & -13.0 & 5.5 & 2.6 & 1.7 & -3.1 & & & \\
\hline \multirow[t]{2}{*}{49} & 0.38 & -1.05 & 0.44 & 0.24 & 0.10 & -0.20 & 0.74 & 4.89 & 0.03 \\
\hline & 4.2 & -12.2 & 3.5 & 3.3 & 1.3 & -4.7 & & & \\
\hline \multirow[t]{2}{*}{50} & 0.35 & -0.91 & 0.38 & 0.16 & 0.12 & -0.18 & 0.73 & 5.22 & 0.02 \\
\hline & 3.9 & -11.7 & 3.4 & 2.3 & 1.8 & -4.5 & & & \\
\hline \multirow[t]{2}{*}{51} & 0.21 & -0.78 & 0.34 & 0.18 & 0.10 & -0.19 & 0.78 & 4.41 & 0.04 \\
\hline & 2.3 & -14.4 & 4.2 & 3.6 & 2.1 & -3.5 & & & \\
\hline \multirow[t]{2}{*}{52} & 0.40 & -0.91 & 0.39 & 0.23 & 0.18 & -0.13 & 0.74 & 1.07 & 0.30 \\
\hline & 4.5 & -11.3 & 3.5 & 3.2 & 2.4 & -4.5 & & & \\
\hline \multirow[t]{2}{*}{53} & 0.29 & -0.91 & 0.43 & 0.11 & 0.15 & -0.13 & 0.76 & 5.22 & 0.02 \\
\hline & 2.8 & -13.6 & 4.1 & 1.7 & 2.5 & -3.5 & & & \\
\hline \multirow[t]{2}{*}{54} & 0.49 & -0.94 & 0.69 & 0.08 & 0.10 & -0.08 & 0.91 & 1.29 & 0.26 \\
\hline & 7.8 & -22.4 & 10.7 & 2.2 & 2.7 & -3.4 & & & \\
\hline \multirow[t]{2}{*}{55} & 0.42 & -0.83 & 0.36 & 0.15 & 0.14 & -0.26 & 0.77 & 2.98 & 0.09 \\
\hline & 5.2 & -10.9 & 3.3 & 2.3 & 2.2 & -5.4 & & & \\
\hline \multirow[t]{2}{*}{57} & 0.27 & -1.08 & 0.60 & 0.31 & 0.09 & -0.11 & 0.82 & 0.95 & 0.33 \\
\hline & 3.2 & -16.7 & 6.0 & 5.3 & 1.4 & -3.7 & & & \\
\hline \multirow[t]{2}{*}{58} & 0.35 & -0.87 & 0.44 & 0.18 & 0.09 & -0.14 & 0.73 & 3.10 & 0.09 \\
\hline & 3.9 & -11.4 & 4.2 & 2.7 & 1.3 & -4.1 & & & \\
\hline
\end{tabular}

Ver notas del cuadro 4.1

En la gráfica 4.2 se presenta un resumen de los resultados anteriormente descritos para las elasticidades de corto plazo en todos los sectores. En el eje horizontal se tiene la elasticidad tipo de cambio. Por lo tanto, puntos debajo de la línea de $45^{\circ}$ indican que (el negativo de) la elasticidad tipo de cambio es mayor que la elasticidad salario, lo que a su vez implica que $\Delta \Psi>0$. 
G R Á F I C A 4.2 Elasticidades estimadas de corto plazo

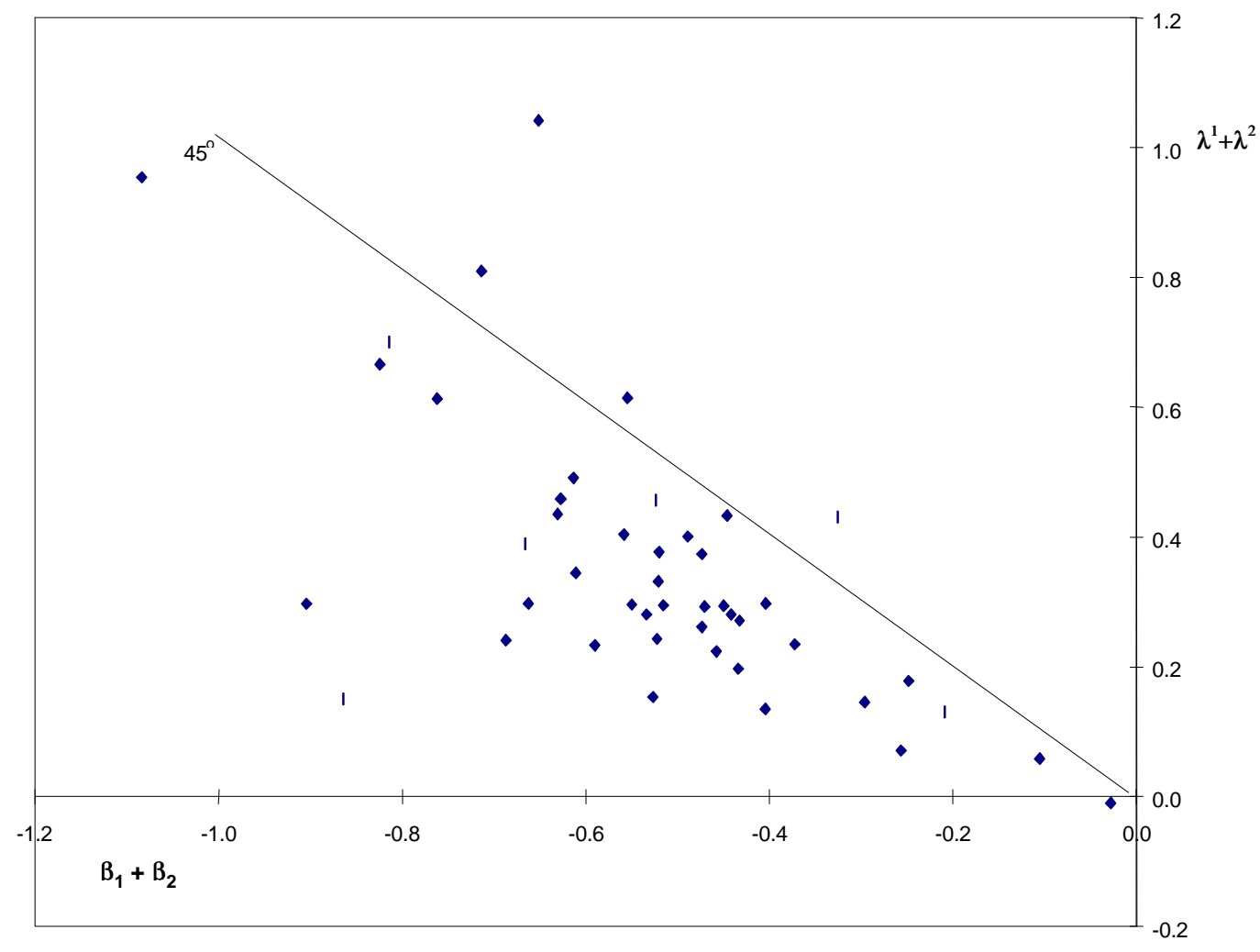

\subsection{Velocidad de Ajuste}

Como se señaló anteriormente, el residual rezagado ajustado, $\varepsilon_{i t-1}$, en el modelo de corrección de errores mide la velocidad de ajuste en respuesta a un desequilibrio. El coeficiente de este término, $\alpha_{1}$, debe ser negativo ya que de lo contrario el modelo nunca se ajustaría al presentarse una desviación del equilibrio.

Si este coeficiente fuera igual a menos 1 el ajuste sería inmediato, mientras que si en valor absoluto se encontrase entre 0 y 1 el ajuste sería gradual para completarse en infinito. El número de periodos $(\tau)$ que transcurren para lograr un cierto porcentaje del ajuste $(\omega)$ se define como:

$$
\tau=\frac{\ln (1-\omega)}{\ln (1-|\alpha|)}
$$


De esta manera, por ejemplo, si $\alpha$ fuera igual (0.5), el cincuenta por ciento del ajuste se da en el primer periodo. Utilizando los resultados presentados en los cuadros 4.1 al 4.4, a continuación se presentan el número de periodos necesarios para alcanzar un determinado porcentaje de ajuste a desequilibrios en cada una de las ramas de la industria manufacturera.

C U A D R O 4.5

Número de periodos necesarios para alcanzar un determinado porcentaje de ajuste

\begin{tabular}{|c|c|c|c|c|c|}
\hline \multirow{2}{*}{ Rama } & \multirow{2}{*}{$|\alpha|$} & \multicolumn{4}{|c|}{ Parcentaje delajuste } \\
\hline & & $50 \%$ & $60 \%$ & $70 \%$ & $80 \%$ \\
\hline 11 & 0.08 & 9 & 11 & 15 & 20 \\
\hline 12 & 0.11 & 6 & 8 & 11 & 14 \\
\hline 13 & 0.21 & 3 & 4 & 6 & 7 \\
\hline 14 & 0.06 & 12 & 15 & 20 & 27 \\
\hline 15 & 0.05 & 14 & 18 & 24 & 32 \\
\hline 16 & 0.14 & 5 & 7 & 8 & 11 \\
\hline 17 & 0.26 & 3 & 4 & 4 & 6 \\
\hline 18 & 0.34 & 2 & 3 & 3 & 4 \\
\hline 19 & 0.10 & 7 & 9 & 12 & 16 \\
\hline 20 & 0.13 & 5 & 7 & 9 & 12 \\
\hline 21 & 0.40 & 2 & 2 & 3 & 4 \\
\hline 22 & 0.12 & 6 & 8 & 10 & 13 \\
\hline 23 & 0.00 & - & - & - & - \\
\hline 24 & 0.23 & 3 & 4 & 5 & 7 \\
\hline 25 & 0.14 & 5 & 7 & 8 & 11 \\
\hline 26 & 0.17 & 4 & 5 & 7 & 9 \\
\hline 27 & 0.12 & 6 & 8 & 10 & 13 \\
\hline 28 & 0.07 & 10 & 13 & 17 & 23 \\
\hline 29 & 0.11 & 6 & 8 & 11 & 14 \\
\hline 30 & 0.17 & 4 & 5 & 7 & 9 \\
\hline 31 & 0.08 & 9 & 11 & 15 & 20 \\
\hline 32 & 0.03 & 23 & 31 & 40 & 53 \\
\hline 33 & 0.18 & 4 & 5 & 7 & 9 \\
\hline 34 & 0.10 & 7 & 9 & 12 & 16 \\
\hline 35 & 0.08 & 9 & 11 & 15 & 20 \\
\hline 36 & 0.25 & 3 & 4 & 5 & 6 \\
\hline 37 & 0.13 & 5 & 7 & 9 & 12 \\
\hline 38 & 0.00 & - & - & - & - \\
\hline 39 & 0.13 & 5 & 7 & 9 & 12 \\
\hline 40 & 0.24 & 3 & 4 & 5 & 6 \\
\hline 41 & 0.15 & 5 & 6 & 8 & 10 \\
\hline 42 & 0.17 & 4 & 5 & 7 & 9 \\
\hline 43 & 0.08 & 9 & 11 & 15 & 20 \\
\hline 44 & 0.06 & 12 & 15 & 20 & 27 \\
\hline 45 & 0.10 & 7 & 9 & 12 & 16 \\
\hline 46 & 0.22 & 3 & 4 & 5 & 7 \\
\hline 47 & 0.16 & 4 & 6 & 7 & 10 \\
\hline 48 & 0.11 & 6 & 8 & 11 & 14 \\
\hline 49 & 0.20 & 4 & 5 & 6 & 8 \\
\hline 50 & 0.18 & 4 & 5 & 7 & 9 \\
\hline 51 & 0.19 & 4 & 5 & 6 & 8 \\
\hline 52 & 0.13 & 5 & 7 & 9 & 12 \\
\hline 53 & 0.13 & 5 & 7 & 9 & 12 \\
\hline 54 & 0.08 & 9 & 11 & 15 & 20 \\
\hline 55 & 0.26 & 3 & 4 & 4 & 6 \\
\hline 57 & 0.11 & 6 & 8 & 11 & 14 \\
\hline 58 & 0.14 & 5 & 7 & 8 & 11 \\
\hline
\end{tabular}




\subsection{Pass-through, concentración de la industria e insumos importados}

El cuadro 4.6 muestra los resultados de probar la especificación (23). Como se esperaba, el diferencial entre las elasticidades salario y tipo de cambio, $\Delta \Psi=\frac{a}{b N p}$, aumenta con la concentración, y es independiente del uso de insumos importados. Este resultado es robusto al incluir sólo aquellas ramas para las cuales $\Delta \Psi$ es estadísticamente positiva.

C U A D R O 4.6

Pass-through a precios en dólares, concentración e insumos importados

Variable dependiente: $\Delta \Psi$

\begin{tabular}{lcc}
\hline & Todas las ramas & $\Delta \Psi$ estadísticamente $>0$ \\
Concentración & $0.40 *$ & 0.39 * \\
& $(0.06)$ & $(0.09)$ \\
& & \\
Ins. importados & 0.05 & 0.25 \\
& $(0.15)$ & $(0.24)$ \\
No. Observ. & 47 & 26 \\
$\mathrm{R}^{2}$ & 0.65 & 0.62 \\
\hline
\end{tabular}

Números en paréntesis son errores estándar.

* Significativo a un nivel de $99 \%$

Los resultados del cuadro 4.7 están también en línea con las predicciones teóricas: el pass-through del tipo de cambio (salarios domésticos) hacia los precios domésticos es significativamente mayor (menor) en sectores que dependen en mayor forma de insumos importados.

C U A D R O 4.7

Pass-through de precios en pesos e insumos importados

\begin{tabular}{lcc}
\hline Variable dependiente: & $\begin{array}{c}\text { Elasticidad de largo plazo del precio al } \\
\text { salario doméstico } \\
\text { tipo de cambio }\end{array}$ & $-0.48^{* *}$ \\
Insumos importados & $0.35^{*}$ & $(0.17)$ \\
& $(0.21)$ & 0.46 \\
Constante & 0.52 & $(0.04)$ \\
& $(0.05)$ & 47 \\
No. Obs. & 47 & 0.13 \\
$\mathrm{R}^{2}$ & 0.04 & \\
\hline
\end{tabular}

** Significativo a un nivel de $99 \%$

* Significativo a un nivel de $90 \%$ 


\section{Conclusiones}

Se ha demostrado que en el corto plazo los movimientos en el tipo de cambio nominal tendrán un mayor efecto sobre los precios domésticos de equilibrio (medidos en unidades de moneda extranjera) que movimientos proporcionales en el salario doméstico nominal. La diferencia entre ambos efectos es mayor al incrementarse la concentración industrial y esta diferencia es independiente del uso de insumos importados por parte de las empresas domésticas. Sin embargo, en el largo plazo este resultado parece desaparecer.

Asimismo, se demostró que el pass-through del tipo de cambio convencional a los precios es mayor para sectores con mayor dependencia de insumos importados, mientras que estos mismos cuentan con un menor pass-through de salarios.

En resumen, las implicaciones principales de este estudio son las siguientes: (1) debemos ser cuidadosos al predecir el comportamiento de los precios de bienes industriales ante movimientos en el tipo de cambio real. Es preciso saber qué variable está impulsando el movimiento de este indicador antes de alcanzar una conclusión. (2) Los esfuerzos para aumentar la competencia industrial reducirán el efecto de los movimientos del tipo de cambio nominal más rápido que el efecto de movimientos en los salarios nominales sobre los precios de equilibrio. (3) El uso de grandes cantidades de insumos importados en el proceso productivo reduce el impacto provocado por aumentos en el salario sobre los precios domésticos. Sin embargo, este hecho hace a los precios más vulnerables a movimientos en el tipo de cambio nominal. 


\section{Apéndice A. Elasticidades precio y markups de equilibrio}

El propósito de este apéndice es analizar las implicaciones de los resultados sobre los markups de equilibrio. Si el tipo de cambio y la elasticidad del salario de los precios domésticos medidos en unidades de moneda doméstica $(p)$ no suman la unidad, entonces los markups no serán homogéneos de grado uno en relación al precio de los factores. Definimos $\mu(E, w, \chi)=\frac{p(E, w, \chi)}{c(E, w)}$ como el markup del precio de equilibrio $(p)$ de la empresa doméstica sobre los costos unitarios $(c)$, donde el término $\chi$ incluye otros parámetros del modelo. Si la demanda del producto final presenta elasticidad constante, como en el modelo de Dixit-Stiglitz que se especifica en el apéndice B, entonces el markup (redefinido como $\frac{p-c}{p}$ ) sería independiente tanto del tipo de cambio como del salario. En general, la elasticidad del markup al tipo de cambio $\left(\Psi_{E}^{\mu}\right)$ y al salario doméstico $\left(\Psi_{w}^{\mu}\right)$ se definen como:

$$
\Psi_{i}^{\mu}=\frac{\partial \mu}{\partial i} \frac{i}{\mu}=\frac{\partial p}{\partial i} \frac{i}{p}-\frac{\partial c}{\partial i} \frac{i}{c}=\Psi_{i}^{p}-\Psi_{i}^{c}
$$

donde $i=E, w$.. Usando la ecuación (5) del texto es fácil demostrar que:

$$
\Psi_{E}^{c}=1-\Psi_{w}^{c}
$$

Combinando (8), (9) y (A2), las elasticidades del markup se pueden reexpresar como:

$$
\begin{gathered}
\Psi_{w}^{\mu}=\Psi_{w}^{p_{e}}-\Psi_{w}^{c} \\
\Psi_{E}^{\mu}=\Psi_{E}^{p}-1+\Psi_{w}^{c}=\Psi_{E}^{p_{e}}+\Psi_{w}^{c}
\end{gathered}
$$

Dado que $\Psi_{E}^{p_{e}}$ es $<0, \Psi_{w}^{p_{e}}>0, \mathrm{y} \Psi_{w}^{c}>0$, el efecto de los movimientos en $E$ y/o $w$ sobre el markup es indeterminado. De cualquier forma, no hay ambigüedad en el efecto sobre el markup causado por los movimientos proporcionales en el tipo de cambio y el salario que mantiene el tipo de cambio real constante. Para demostrar lo anterior, se hace referencia a la ya antes vista relación entre el tipo de cambio y la elasticidad del salario dada por: 


$$
\Delta \Psi=-\Psi_{E}^{p_{e}}-\Psi_{w}^{p_{e}}
$$

Si $\hat{E}=\hat{w}=g$ entonces los costos unitarios también cambiarán en $\mathrm{g}$ (ya que el costo unitario es linealmente homogéneo a los precios de los factores), pero el TCR e mantendrá constante. Usando las ecuaciones (A3) a (A5), el cambio en el markup está dado por:

$$
\hat{\mu}=g\left(\Psi_{E}^{p_{e}}+\Psi_{w}^{p_{e}}\right)=-g \Delta \Psi
$$

Si $\Delta \Psi$ es positivo, como en el planteamiento de Cournot, los cambios en el markup serán en dirección opuesta al signo de $g$. Por ejemplo, una depreciación nominal en el tipo de cambio combinada con un aumento proporcional en el salario doméstico (e.g. $g$ sea positiva) se traduce en un markup menor de equilibrio. Así, el markup no es homogéneo de grado uno con respecto al precio de los factores. 


\section{Apéndice B. Equilibrio de Dixit-Stiglitz (DS)}

En esta sección se presenta el modelo estándar de Dixit y Stiglitz (1977) extendido para permitir que las empresas domésticas usen insumos extranjeros. El principal propósito de este modelo es demostrar la consistencia de los resultados ante demandas diferentes y especificaciones tecnológicas. La característica más importante del modelo DS (en términos de este artículo) es el hecho de que, en contraste con el planteamiento de Cournot presentado en el texto, la demanda por el producto final presenta las propiedades de aquellas con elasticidad constante. En primer lugar se resolverá el modelo extendido DS manteniendo el supuesto de una tecnología de tipo Leontief que se utilizó para resolver el modelo de Cournot. En segundo término, se realizará el mismo análisis sin poner ninguna restricción a la tecnología.

Se asumió que el consumidor representativo tiene un ingreso unitario y consume $\mathrm{n}$ bienes producidos por empresas domésticas y $n *$ bienes producidos por empresas extranjeras. Así, el problema es:

$$
\begin{gathered}
\max \mathrm{U}=\left[\sum_{j=1}^{n+n^{*}} X_{j}^{\alpha}\right]^{\frac{1}{\alpha}} \\
\text { s.a. } \quad \sum_{j=1}^{n+n^{*}} p_{j} X_{j}=1
\end{gathered}
$$

donde $0<\alpha<1$ para asegurar concavidad.

Usando la identidad anterior junto con la restricción presupuestal y definiendo el nivel de precios industriales $\operatorname{como}^{33} P=\left[\frac{1}{N} \sum_{j} p_{j}^{\frac{\alpha}{\alpha-1}}\right]^{\frac{\alpha-1}{\alpha}}$ donde $N=n+n^{*}$ y $\sigma=\frac{1}{1-\alpha}$ es la elasticidad sustitución constante entre cualesquiera dos bienes, se puede encontrar una función de demanda para cada bien del conjunto $N$ :

$$
X_{i}\left(p_{i}, P\right)=\frac{p_{i}^{-\sigma} P^{\sigma-1}}{N}
$$

\footnotetext{
33 Esta definición tiene las propiedades típicas de un nivel de precios, como: homogeneidad de grado uno en precios y derivadas parciales positivas con respecto a todos sus argumentos.
} 
$\mathrm{Si}$ se supone que cada empresa es lo suficientemente pequeña de manera que alteraciones en su propio precio no modificarán el índice de precios industriales, la elasticidad precio (constante) de la demanda para cada bien está dada por:

$$
\frac{\partial X_{i}}{\partial p_{i}} \frac{p_{i}}{X_{i}}=-\sigma \quad \forall i
$$

Sustituyendo (A7) en las funciones de beneficios y maximizando, se obtiene el precio de equilibrio de las empresas representativas domésticas y extranjeras. Estos resultados están dados respectivamente por:

$$
\begin{aligned}
& p^{d}=\frac{c(w, E)}{\alpha} \\
& p^{f}=\frac{c^{*} E}{\alpha}
\end{aligned}
$$

donde, si las empresas usaran una tecnología de tipo Leontief, $c(w, E)=E \gamma+w(1-\gamma)$, y $c *$ 1 (ver ecuaciones (1) a (6) en el texto). Se puede usar (A9) y (A10) para encontrar el nivel agregado de precios de equilibrio y la cantidad producida por cada empresa:

$$
\begin{aligned}
P(\omega, E) & =\left[\frac{1}{N}\left(n\left(\frac{c}{\alpha}\right)^{1-\sigma}+n *\left(\frac{E}{\alpha}\right)^{1-\sigma}\right)\right]^{\frac{1}{1-\sigma}} \\
X_{i}(\omega, E) & =\frac{1}{N}\left(\frac{c_{i}}{\alpha}\right)^{-\sigma}\left[\frac{1}{N}\left(n\left(\frac{c}{\alpha}\right)^{1-\sigma}+n *\left(\frac{E}{\alpha}\right)^{1-\sigma}\right)\right]^{-1}
\end{aligned}
$$

En base a (A11), se puede demostrar que el nivel de precios medido en unidades de moneda extranjera $\left(p^{\mathrm{e}}\right)$ no varía ante movimientos proporcionales de tipo de cambio y salarios. Formalmente, bajo este escenario se obtiene que:

$$
\Psi_{w}^{p_{e}}=-\Psi_{E}^{p_{e}}
$$


que a su vez implica que $\Delta \Psi=0$. Esto significa que cambios proporcionales en los salarios y en el tipo de cambio no afectan el nivel agregado de precios medido en unidades de moneda extranjera. Si se analiza el precio de equilibrio de las empresas representativas nacionales medido en moneda doméstica $\left(p^{d}\right)$, se puede demostrar que $\Psi_{E}^{p^{d}}=\frac{\gamma E}{c_{i}}, \quad$ y $\quad \Psi_{w}^{p^{d}}=\frac{(1-\gamma) w}{c_{i}}$. Como con el nivel agregado de precios, se tiene que $\Delta \Psi^{d}=1-\Psi_{E}^{p^{d}}-\Psi_{w}^{p^{d}}=0$. De cualquier forma, al contrario del modelo estándar $\mathrm{DS}^{34}$, bajo el planteamiento extendido el uso de insumos extranjeros se traduce en un pass-through incompleto de los salarios domésticos a los precios domésticos ${ }^{35}$.

¿Qué sucede al relajar el supuesto de una tecnología Leontief mientras se mantiene una función de demanda con elasticidad constante? Considere el caso del típico bien doméstico, donde el precio de equilibrio está dado por la ecuación (9). Bajo una función de costos unitarios de la forma:

$$
\Omega=c(w, E)+h
$$

donde $h$ es un costo fijo por unidad de producto, las elasticidades precio (medidas en unidades de moneda extranjera) con respecto al tipo de cambio y al salario están dadas respectivamente por:

$$
\begin{aligned}
\frac{\partial p^{d}}{\partial E} \frac{E}{p^{d}}-1 & =\frac{1}{p^{d} \alpha}\left[\frac{\partial c}{\partial E} E-\Omega\right] \\
\frac{\partial p^{d}}{\partial w} \frac{w}{p^{d}} & =\frac{\partial c}{\partial w} \frac{w}{p^{d} \alpha}
\end{aligned}
$$

El diferencial ahora está dado por:

$$
\Delta \Psi^{d}=\frac{1}{p^{d} \alpha}\left[c(w, E)+h-\frac{\partial c}{\partial E} E-\frac{\partial c}{\partial w} w\right]
$$

\footnotetext{
34 Ver Dornbusch (1987)

35 El passthraugh del tipo de cambio a los precios de las empresas extranjeras se mantiene de forma completa (igual a uno).
} 
Sin tomar en cuenta el tipo de tecnología que se tenga, si la empresa busca la minimización de costos al nivel de precios dado, se puede demostrar que $c(w, E)$ es homogénea de grado uno. En base a lo anterior, (A17) puede reexpresarse como:

$$
\Delta \Psi^{d}=\frac{h}{\Omega}=\frac{h}{c(w, E)+h}
$$

Así, la existencia de costos fijos apoya que $\Delta \Psi>0$ aun en presencia de la función de demanda con elasticidad precio constante. 


\section{Apéndice C. Sectores de la Industria Manufacturera en México, Fuentes, Construcción y}

\section{Descripción de los Datos.}

\begin{tabular}{|c|c|}
\hline \multicolumn{2}{|c|}{ RAMA } \\
\hline \multicolumn{2}{|c|}{ División 1: Productos alimenticios, bebidas y tabaco } \\
\hline 11 & Productos cárnicos y lácteos \\
\hline 12 & Envasado de frutas y legumbres \\
\hline 13 & Molienda de trigo y sus productos \\
\hline 14 & Molienda de nixtamal y sus productos \\
\hline 15 & Procesamiento de café \\
\hline 16 & Azúcar y sus derivados \\
\hline 17 & Aceites y grasas vegetales comestibles \\
\hline 18 & Alimentos para animales \\
\hline 19 & Otros productos alimenticios \\
\hline 20 & Bebidas alcohólicas \\
\hline 21 & Cerveza \\
\hline 22 & Refrescos y bebidas embotelladas \\
\hline 23 & Tabaco y sus productos \\
\hline \multicolumn{2}{|c|}{ División 2: Textiles, prendas de vestir e industria del cuero } \\
\hline 24 & Hilados y tejidos de fibras blandas \\
\hline 25 & Hilados y tejidos de fibras duras \\
\hline 26 & Otras industrias textiles \\
\hline 27 & Prendas de vestir \\
\hline 28 & Cuero y sus productos \\
\hline \multicolumn{2}{|c|}{ División 3: Industria de la madera y productos de madera } \\
\hline 29 & Aserraderos incluso triplay \\
\hline 30 & Otras industrias de la madera \\
\hline \multicolumn{2}{|c|}{ División 4: Papel, productos de papel, imprentas y editoriales } \\
\hline 31 & Papel y cartón \\
\hline 32 & Imprentas y editoriales \\
\hline \multicolumn{2}{|c|}{ División 5: Sustancias químicas derivadas del petróleo, caucho y plásticos } \\
\hline 33 & Refinación de petróleo \\
\hline 34 & Petroquímica básica \\
\hline 35 & Química básica \\
\hline 36 & Abonos y fertilizantes \\
\hline 37 & Resinas sintéticas, plástico y fibras \\
\hline 38 & Productos farmacéuticos \\
\hline 39 & Jabones, detergentes, perfumes y cosméticos \\
\hline 40 & Otras industrias químicas \\
\hline 41 & Productos de hule \\
\hline 42 & Productos de plástico \\
\hline \multicolumn{2}{|c|}{ División 6: Productos de minerales no metálicos excepto petróleo y carbón } \\
\hline 43 & Vidrio y sus productos \\
\hline 44 & Cemento \\
\hline 45 & Otros productos de minerales no metálicos \\
\hline \multicolumn{2}{|c|}{ División 7: Industrias metálicas básicas } \\
\hline 46 & Industrias básicas de hierro y acero \\
\hline 47 & Industrias básicas de metales no ferrosos \\
\hline \multicolumn{2}{|c|}{ División 8: Productos metálicos, maquinaria y equipo } \\
\hline 48 & Muebles y accesorios metálicos \\
\hline 49 & Productos metálicos estructurares \\
\hline 50 & Otros productos metálicos \\
\hline 51 & Maquinaria y equipos no eléctricos \\
\hline 52 & Maquinaria y aparatos eléctricos \\
\hline 53 & Aparatos electrodomésticos \\
\hline 54 & Equipos y accesorios electrónicos \\
\hline 55 & Equipos y aparatos eléctricos \\
\hline 56 & Vehículos y automóviles \\
\hline 57 & Carrocerías y partes automotrices \\
\hline 58 & Otros equipos y materiales de transporte \\
\hline
\end{tabular}

Los precios industriales considerados $(p)$ son precios de consumo publicados en los Indicadores Económicos del Banco de México. Dado que el comercio internacional de México se enfoca básicamente al mercado de EE.UU., la definición de tipo de cambio será la paridad peso/dólar que se 
usa en las transacciones internacionales, como la publica el Banco de México. Las series de salario doméstico $(w)$ se definen como el cociente que se deriva del total de pagos salariales entre el empleo total, como se reporta en una encuesta mensual del sector manufacturero dirigida por el Instituto Nacional de Estadística Geografía e Informática (INEGI). Estas series están desagregadas en subdivisiones de manera que se tienen ocho series en total.

La razón de concentración se calcula a partir de los resultados de un estudio realizado por el INEGI en 1993. Es un promedio ponderado de las razones de concentración de tres empresas de cada sector manufacturero $^{36}$. La encuesta considera a empresas tanto con capital en moneda extranjera como sin él.

Para construir el estimado del uso específico por sector de insumos importados en cualquier momento del tiempo $\left(\gamma_{\mathrm{it}}\right)$, se partió de la siguiente definición:

$$
\gamma_{i t}=\frac{R^{*}{ }_{i t}}{L_{i t}+R^{*}{ }_{i t}}=\frac{M_{i t}}{Y_{i t}} \frac{P_{i t}}{E_{t} P^{*}{ }_{i t}}
$$

donde $R^{*}$ denota unidades de insumos importados, $L$ son unidades de trabajo doméstico, $\mathrm{M}$ es el valor nominal de las importaciones en pesos $\left(R * \cdot P^{*} \cdot E\right)$, e $\mathrm{Y}$ es el ingreso nominal en pesos $((L+R) \cdot P)$

En México no se publican datos sobre el nivel de importaciones de las empresas. Para resolver esta limitante se construyó una lista de 50 grandes empresas para cada una de las ramas de acuerdo al total de activos en 1993 a partir de una base de datos que la Secretaría del Hacienda mantiene con fines fiscales. Esta base de datos contiene una identificación fiscal que es también utilizada al importar mercancía extranjera a través de un agente aduanal. La identificación fue introducida en la computadora de la autoridad aduanera ${ }^{37}$ para encontrar el valor agregado por sector de las importaciones en dólares corrientes de estas 50 empresas, trimestralmente, desde 1991 hasta junio de 1996. Esta fuente clasifica las importaciones según su tipo en bienes de consumo, bienes intermedios o bienes de capital. Al agregar el total de los bienes importados intermedios y de capital y multiplicar

36 Para cada sector, el denominador de la razón de concentración es la producción total del sector. Una medida más precisa, también tomaría en cuenta las importaciones.

${ }^{37}$ Esta es la fuente que utiliza el Banco de México para construir las estadísticas de balanza comercial. 
el resultado por el tipo de cambio promedio durante el trimestre, obtenemos una estimación de $M$. La producción trimestral nominal por sector se construyó usando el índice de la producción industrial por sector y tomando el PIB nominal de 1990 de cada sector como base (la fuente de las dos variables es el INEGI). Los precios $(P)$ utilizados para cada sector se obtuvieron de la fuente descrita anteriormente y $P^{*}$ es el índice de precios al productor de bienes industriales de EE.UU., obtenido del International Financial Statistics (IFS). Por último, el uso específico de insumos importados por rama para toda la muestra se define como el promedio aritmético de $\gamma_{\mathrm{it}}{ }^{38}$.

${ }^{38}$ Se consideraron 22 observaciones para cada sector (trimestrales desde enero de 1990 hasta junio de 1996) 


\section{Referencias}

Aspe, P. (1993), “Economic Transformation the Mexican Way”, Cambridge, Mass., MIT Press.

Conesa, A. (1997), "Exchange Rate Risk and the Debt Portfolio Composition of Mexican Firms", mimeo, MIT.

Dixit, A, and Stiglitz, J. (1977), "Monopolistic Competition and Optimum Product Diversity". American Economic Review ,67 (Junio).

Dixit, A. (1986), "Comparative Statics for Oligopoly". International Economic Review, 27 (Febrero).

Dornbusch, R. (1987), “Exchange Rates and Prices”. American Economic Review 77 (Marzo), no1.

Dornbusch, R., and Werner, A. (1994), “Mexico: Stabilization, Reform, and No Growth”, Brookings Papers on Economic Activity, 1:1994.

Dornbusch, R., Goldfajn, I., and Valdes, R. (1995), "Currency Crises and Collapses", Brookings Papers on Economic Activity, 2:1995.

Dwyer, J., Kent, C., and Pease A. (1994). "Exchange Rate Pass-Through: Testing the Small Country Assumption for Australia”. The Economic Record, vol.70, No.211 (Diciembre).

Estadísticas Financieras Internacionales, Fondo Monetario Internacional, varios tomos.

Gonzalez, J.A. (1996), "Trade Regimes and Exchange Rate Pass-Through: Is there a Mexican Puzzle", Mimeo, Harvard University.

Hamilton, J.D. (1994), “Time Series Analysis”. Princeton University Press.

Indicadores Económicos, Banco de México, Dirección de Investigación Económica., varios tomos.

Instituto Nacional de Estadistica, Geografía e Informática (INEGI), (1994), XIV Censo Industrial, Censos Económicos.

Kremers, J. J. M., Ericsson, N.R. and Dolado, J.J. (1992). "The Power of Cointegration Tests". Oxford Bulletin of Economics and Statistics 54.

Salop, S. (1979). Monopolistic Competition with Outside Goods”. Bell Journal of Economics 10.

Shaked, A., and Sutton, J. (1982), "Relaxing Price Competition through Product Differentiation". Review of Economic Studies 49. 
Varian, H. (1992), “Microeconomic Analysis”, W.W. Norton \& Company Press. 